\title{
A tumor microenvironment-specific gene expression signature predicts chemotherapy resistance in colorectal cancer patients
}

\author{
Xiaoqiang Zhu ${ }^{1,2,7}$, Xianglong Tian ${ }^{1,3,7}$, Linhua $\mathrm{Ji}^{4,7}$, Xinyu Zhang ${ }^{1}$, Yingying $\mathrm{CaO}^{1}$, Chaoqin Shen ${ }^{1}$, Ye Hu $\mathrm{H}^{5,6}$, Jason W. H. Wong $\mathbb{1}^{2}$,
} Jing-Yuan Fang $\mathbb{1}^{1 凶}$, Jie Hong $^{1 凶}$ and Haoyan Chen ${ }^{1 凶}$

Studies have shown that tumor microenvironment (TME) might affect drug sensitivity and the classification of colorectal cancer (CRC). Using TME-specific gene signature to identify CRC subtypes with distinctive clinical relevance has not yet been tested. A total of 18 "bulk" RNA-seq datasets (total $n=2269$ ) and four single-cell RNA-seq datasets were included in this study. We constructed a "Signature associated with FOLFIRI resistant and Microenvironment" (SFM) that could discriminate both TME and drug sensitivity. Further, SFM subtypes were identified using $K$-means clustering and verified in three independent cohorts. Nearest template prediction algorithm was used to predict drug response. TME estimation was performed by CIBERSORT and microenvironment cell populations-counter (MCP-counter) methods. We identified six SFM subtypes based on SFM signature that discriminated both TME and drug sensitivity. The SFM subtypes were associated with distinct clinicopathological, molecular and phenotypic characteristics, specific enrichments of gene signatures, signaling pathways, prognosis, gut microbiome patterns, and tumor lymphocytes infiltration. Among them, SFM-C and $-\mathrm{F}$ were immune suppressive. SFM-F had higher stromal fraction with epithelial-tomesenchymal transition phenotype, while SFM-C was characterized as microsatellite instability phenotype which was responsive to immunotherapy. SFM-D, $-E$, and $-F$ were sensitive to FOLFIRI and FOLFOX, while SFM-A, $-B$, and $-C$ were responsive to EGFR inhibitors. Finally, SFM subtypes had strong prognostic value in which SFM-E and -F had worse survival than other subtypes. SFM subtypes enable the stratification of CRC with potential chemotherapy response thereby providing more precise therapeutic options for these patients.

npj Precision Oncology (2021)5:7; https://doi.org/10.1038/s41698-021-00142-x

\section{INTRODUCTION}

Colorectal cancer (CRC) is a disease with great heterogeneity characterized as distinctive molecular pathogenesis, histogenesis, and drug sensitivity ${ }^{1,2}$. The heterogeneity of CRC has been revealed by using whole-genome sequencing (WGS), epigenetic analysis, and gene expression profiles. For instance, at the genetic level, some DNA markers have been recognized including microsatellite instability (MSI), CpG island methylator phenotype (CIMP), chromosomal instability (CIN), BRAF, and KRAS mutations. Further, tumor microenvironment (TME) components consist of distinctive and interacting cell populations, including tumor epithelial cells, immune cells, and cancer-associated fibroblasts $(\mathrm{CAFs})^{3,4}$. The diversity of TME has made it possible to perform immune classification of cancers regarding prognosis ${ }^{5}$, chemotherapy $^{6}$, and immunotherapy ${ }^{7}$ response prediction. For example, MSI tumor displays higher densities of type $1 \mathrm{~T}$ helper, effector memory $\mathrm{T}$ cells ${ }^{8}$, and has good prognosis. It has also shown significant benefit from immune checkpoint blockade therapy, anti-PD1/PDL1 (refs. ${ }^{9,10}$ ). Besides, increasing evidence indicated that the dysbiosis of gut microbiota can lead to the development and progression of CRC by inducting chronic inflammatory state and immune response, regulating stem cell dynamics, producing toxic and genotoxic metabolites, and affecting the host metabolism ${ }^{11}$. Microbial communities also varied in different parts of gut, including distal colon and proximal ileum, both of which admittedly have distinctive prognosis and treatment strategies ${ }^{12}$.

Gene expression-based classifying has shown high efficiency for cancer classification. Several molecular classifiers in CRC have been built using global gene expression analysis, including colon cancer subtypes $(\mathrm{CCS})^{13}$, the Colorectal Cancer Assigner ${ }^{14}$, colon cancer molecular subtype systems, and colorectal cancer subtyping consortium (CRCSC) ${ }^{15}$. Most of them were constructed directly based on global expression profiles using unsupervised consensus-based clustering algorithms. However, these transcriptome signals derived from both cancer cells and noncancerous components. And these approaches can't distinguish these signals automatically when applied to classification. Recent studies have consistently suggested that TME components played important roles in defining CRC with poor prognosis and immune escape ${ }^{16-18}$. Given that TME also makes great contribution to chemotherapy and immunotherapy sensitivity $7,19,20$, and FOLFIRI (combination of folinic acid, fluorouracil, and irinotecan) and FOLFOX (combination of 5-fluorououracil, leukovorin, and oxaliplatin) are two of the most common first-line treatment strategies for metastatic CRC (mCRC), we proposed that using gene expression profiles that could discriminate both TME and drug sensitivity might be an effective way to define CRC molecular subtypes.

\footnotetext{
${ }^{1}$ State Key Laboratory for Oncogenes and Related Genes, Division of Gastroenterology and Hepatology, Key Laboratory of Gastroenterology and Hepatology, Ministry of Health, Shanghai Institute of Digestive Disease, Renji Hospital, School of Medicine, Shanghai Jiao Tong University, Shanghai, China. ${ }^{2}$ School of Biomedical Sciences, Li Ka Shing Faculty of Medicine, The University of Hong Kong, Pokfulam, Hong Kong SAR, China. ${ }^{3}$ Department of Gastroenterology, Tongren Hospital, Shanghai Jiao Tong University School of Medicine, Shanghai, China. ${ }^{4}$ Department of Gastrointestinal Surgery, Renji Hospital, School of Medicine, Shanghai Jiao Tong University, Shanghai, China. ${ }^{5}$ Department of Gastroenterology, Xinhua Hospital, Shanghai Jiao Tong University School of Medicine, Shanghai, China. 'Women's Cancer Program at the Samuel Oschin Comprehensive Cancer Institute, Cedars-Sinai

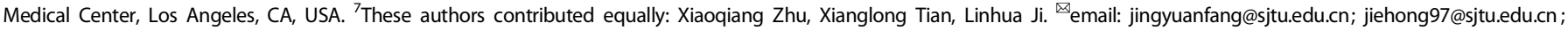
haoyanchen@sjtu.edu.cn
} 
To test this hypothesis, a total of 2269 gene expression profiles from 18 datasets and another four single-cell RNA-sequencing (scRNA-seq) datasets were analyzed in this study to (i) construct a gene signature consisted of genes that could discriminate both TME and drug sensitivity; (ii) further identify a robust molecular classification using this gene signature; and (iii) evaluate the associations between CRC subtypes and clinicopathological factors, common oncogenic mutations, genetic changes, signaling pathways, drug sensitivity, immune infiltration, prognosis, and gut microbiota patterns.

\section{RESULTS}

\section{A chemotherapy resistant gene signature associated with TME}

A total of 2269 bulk gene expression profiles of CRC patients were included in this study (Supplementary Table. 1). Supplementary Figure 1a summarized the schematic workflow of this study. We identified 896 probe sets that were involved with FOLFIRI response. To further select genes that differed from TME, we further identified genes with significantly discriminative expression amongst TME components, such as tumor epithelial cells, immune cells, and stromal cells (see "Methods" section for details). After overlapping the differential probes, we acquired a list of 317 probes which corresponded to 250 unique genes (Fig. 1a, Supplementary Fig. 1b, and Supplementary Table 2) and referred to this gene signature as the "Signature associated with FOLFIRI resistant and Microenvironment" (SFM). To confirm that SFM signature could discriminate TME, we applied SFM signature to four scRNA-seq datasets of human CRC, head and neck squamous cell carcinoma (HNSCC), melanoma and breast cancer (BRCA). Each data contained malignant and nonmalignant cells derived from human tumors, thus enabling to validate the ability of SFM to define the heterogeneity of TME. We explored the global structure of SFM expression in these four SCRNA-seq datasets using $t$ distributed stochastic neighbor embedding ( $t$-SNE). $t$-SNE plot indicated that SFM formed distinct clusters corresponding to different cells types in all the four scRNA-seq datasets, implying the universal ability of SFM to discriminate TME (Fig. 1b-e). SFM expression profiles and SFM gene signature scores further confirmed our observation (Supplementary Fig. 1c-g). Moreover, we found that malignant cells from different origins (i.e., from which tumor patients) could also form distinct clusters, while nonmalignant cells could cluster together regardless of the origins indicating that SFM expression of normal cells had no strong interpatient heterogeneity. Next, we evaluated overlaps among nine published gene signatures with SFM and found large overlaps among some of these gene signatures (Fig. If and Supplementary Table. 3). Intriguingly, the SFM displayed very limited overlaps with other gene signatures. A total of 216 out of the SFM genes were unique; 20, 8, and 6 genes were shared with other gene signatures for one, two, and three times, respectively (Fig. 1g).

\section{K-means clustering CRC subtypes}

To test if SFM could classify CRC subtypes, we used $k$-means clustering algorithm to the classification using the SFM in discovery dataset (GSE39582). By doing so, six subtypes were identified and referred as CRC SFM subtypes from SFM-A to SFM-F (Supplementary Fig. $1 \mathrm{~h}$ and Supplementary Table. 4). The robustness of SFM classification was further validated in two large cohorts and our Renji cohort (Supplementary Fig. 1i-k and Supplementary Table. 4). In Renji cohort, only four SFM subtypes were classified mainly because of small number of sample size. Overall, the proportion of each subtype was similar in three large datasets (Fig. 1h). Given that stromal signal strongly affects the transcriptional classification ${ }^{21}$ and SFM consists of genes predominately expressed in stomal content, we additionally applied
SFM to a patient-derived xenografts CRC dataset (GSE76402, $n=$ $515)$, in which the stromal components from the original tumors have been substituted by murine counterparts ${ }^{21}$. As expected, the SFM classification was not perfect since the SFM genes were not clearly discriminative amongst resulted clusters (Supplementary Fig. 1I). This implied that the SFM classification was also depended on original TME components. After combining the subtype information, we saw that SFM-A (23\%), SFM-B (23\%), and SFM-E (25\%) accounted for larger proportions followed by SFM-C (12\%), SFM-F (10\%), and SFM-D (8\%). We then compared the overlap of SFM subtypes with published CRC classifiers. For each classifier, we combined all used samples that were annotated with individual subtype information. As expected, we saw that SFM subtypes had strong overlaps with other classifiers (Supplementary Fig. 1m). For instance, compared to the CMS subtypes developed by Guinney et al. ${ }^{15}, 61 \%$ of SFM-A and $80 \%$ of SFM-B were CMS2; $81 \%$ of SFMC were $\mathrm{CMS1}$; $33 \%$ of SFM- 1 were SFM-C; half of SFM-D and E, and $92 \%$ of SFM-F were CMS4. As for CCS classification developed by De. Sousa et al. ${ }^{13}$, almost SFM-A (96\%) and SFM-B (75\%) belonged to CCS1; $86 \%$ of SFM-C belonged to CCS2; and all of SFM-F were CCS3. Some overlaps could also be observed for the remaining subtypes including The Cancer Genome Atlas (TCGA) CRC molecular subtypes ${ }^{22}$, C1-C6 clusters developed by Marisa et al. ${ }^{23}$, CRISA-CRISE clusters by Isella et al. ${ }^{21}$, and five molecular subtypes by Sadanandam et al. ${ }^{14}$.

\section{Leading peculiarities of SFM subtypes}

Further analysis implied that SFM subtypes were associated with distinct clinicopathological, molecular and phenotypic characteristics, and specific enrichments of gene signatures and signaling pathways. Firstly, we found higher proportion of stage II and III in each SFM cluster compared to stage I and IV (Fig. 1i). Higher proportion of stage IV could be observed in SFM-E (18\%) and SFMF (17\%). Secondly, $74 \%$ of SFM-C belonged to MSI tumors and $60 \%$ MSI tumors were assigned to SFM-C $(P<0.0001$, Supplementary Fig. 1n). Furthermore, SFM-C and SFM-F were mainly endowed with hypermutation genotype $(P<0.0001$, Supplementary Fig. $1 \mathrm{n})$, proximal colon tumors $(P<0.0001$, Supplementary Fig. $1 \mathrm{n})$. On the contrary, tumors classified as SFM-A-B-D-E showed CIN $+(P<$ 0.0001 , Supplementary Fig. 1n), MSS, non-hypermutant features. In addition to SFM-C, SFM-F was the second subtype enriched with BRAF mutation $(P<0.0001$, Supplementary Fig. $1 \mathrm{n})$. And TP53 was more frequently mutant in SFM-B and SFM-D $(P=0.0034$, Supplementary Fig. 1n), while KRAS mutations were more frequently occurred in SFM-A $(P=0.0001$, Supplementary Fig. $1 \mathrm{n})$. As for oncogenic mutation, we further focused on 95 driver mutations of CRC in TCGA dataset ${ }^{24}$. Among these 95 drivers, SFMC (33\%, 26 in 80$)$ and SFM-D $(21 \%, 5$ in 24$)$ had higher proportion of samples that had more than seven mutant driver genes (Supplementary Fig. 10). In addition, 53 of 95 gene mutation status had significant differences among SFM subtypes $(P<0.05$, Supplementary Fig. 1p).

We used previously reported gene signatures to identify the cell and precursor origins of SFM subtypes based on the nearest template prediction (NTP) algorithm ${ }^{25}$ (Supplementary Fig. 1q, and Supplementary Tables 5 and 6). We applied an intestinal stem cell signature and a colon crypt signature to the four gene expression profiles. SFM-E and SFM-F were found remarkably enriched for "stem-like" phenotype $(P<0.0001)$ and SFM-B-E-F had hallmarks of colon base crypt $(P<0.0001)$. As epithelial-tomesenchymal transition (EMT) has been regarded as a critical process in CRC progression ${ }^{26}$, we applied an EMT signature to our data similarly. The results indicated that SFM-D-E-F were enriched for EMT phenotype $(P<0.0001)$. Besides, serrated CRC is morphologically distinctive compared with conventional CRC and has been suggested to be involved with serrated neoplasia procedure $^{27}$. We found that SFM-C-E-F were characterized as 
a

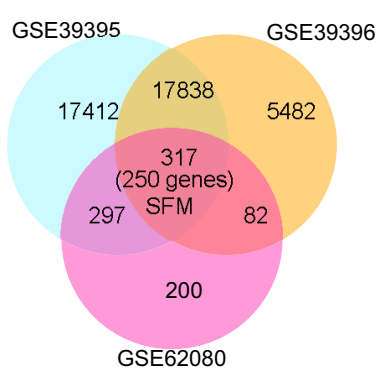

d

GSE72056. melanoma $(n=4054)$

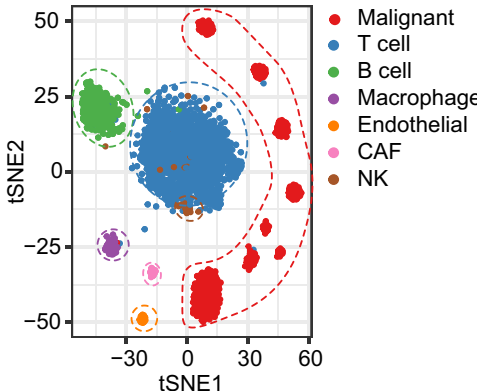

g

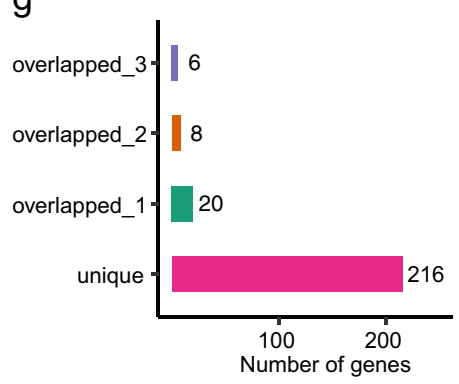

b

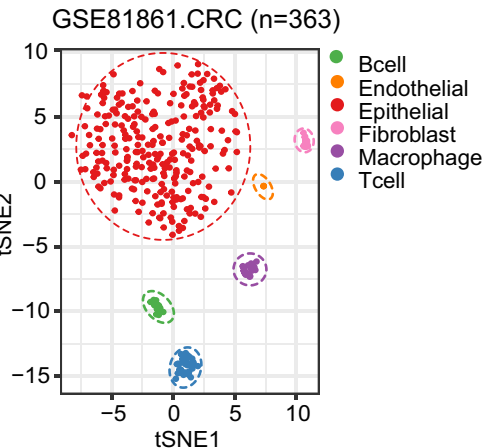

C

GSE103322.HNSCC $(n=5502)$

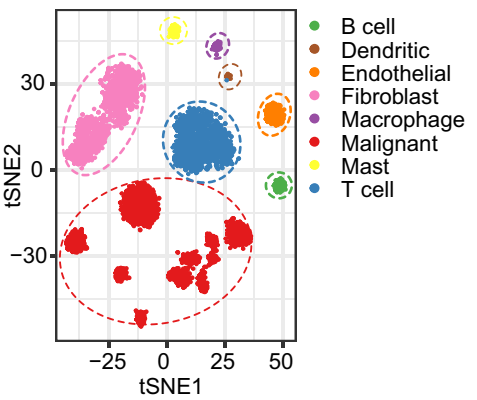

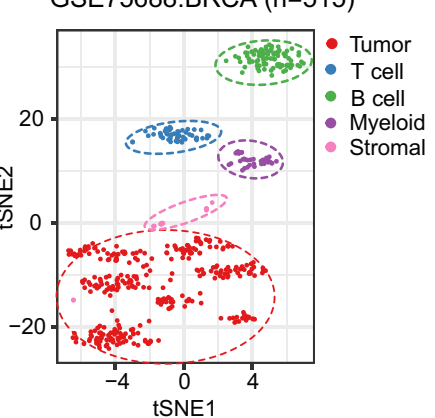

h

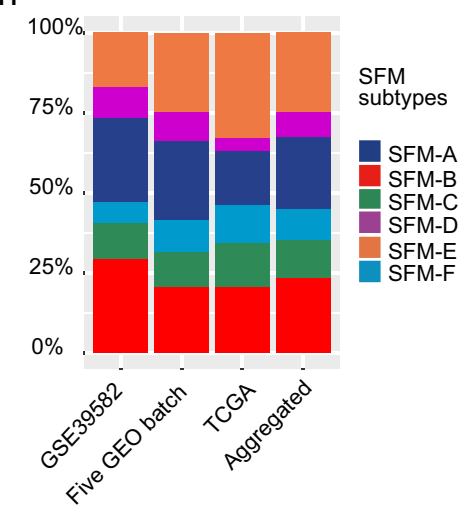

f

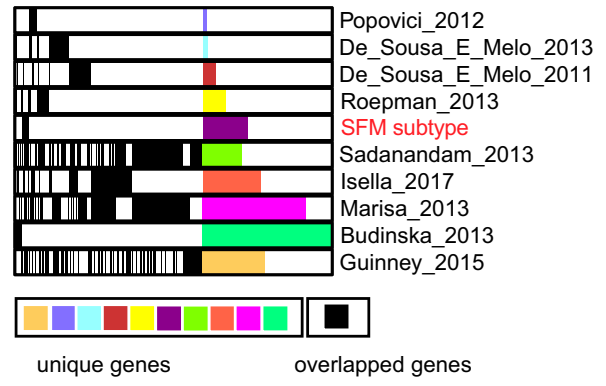

i

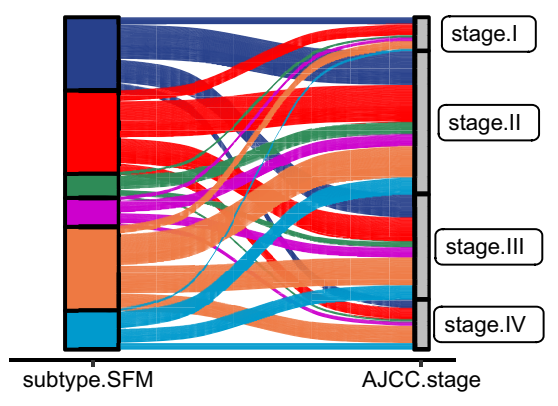

Fig. 1 Construction of SFM signature and subtypes. a Venn diagram of differentially expressed probe sets in three datasets showing intersection of 317 probes (250 unique genes). b-e $t$-SNE plot of SFM gene expression profiles from four datasets (b, CRC, $n=363$; $\mathbf{c}$, HNSCC, $n=5502$; d, melanoma, $n=4054 ; \mathbf{e}$ BRCA, $n=515)$. $\mathbf{f}-\mathbf{g}$ Genes overlapped between SFM and published gene signatures. Each column indicates each gene signature and each row indicate each gene. Shared genes are indicated with black lines (f). Graph depicts the quantification of overlap across these signatures (g). $\mathbf{h}$ Proportion of SFM subtypes in individual and aggregated datasets. $\mathbf{i}$ Sankey diagram showing how each SFM subtype contributes to AJCC stage classification.

"serrated CRC" phenotype, whereas SFM-A-B-D displayed a "conventional CRC" phenotype $(P<0.0001)$.

We also analyzed dysregulated signaling pathways in each SFM subtype. Two thousand top up- and down-expressed genes in each SFM subtype were subjected to perform analysis (Supplementary Table. 7). Most of SFM subtypes were enriched in specific activated pathways (Supplementary Fig. 1r). Specifically, glucose metabolism was activated in SFM-A, while most signaling pathways were downregulated in this subtype. SFM-B showed upregulation of DNA replication/damage associated pathways. Upregulated interleukin-6/8 (IL-6/8) and downregulated EMT and fibroblast growth factor receptor associated pathways were found in SFM-C. SFM-D-E-F all displayed upregulation of cell focal adhesion, collagen formation, and integrin pathways. In SFM-D, IL2 and IL-3 associated pathways were activated, while IL-5 mediated pathway was upregulated in SFM-E. SFM-E and SFM-F displayed significantly upregulated immune system and EMT pathways.

\section{SFM subtype predicted chemotherapy response}

Since SFM signature was correlated with FOLFIRI sensitivity derived from GSE62080, we first performed $K$-means clustering using the SFM signature in GSE62080 dataset to examine whether SFM subtypes were associated with drug response. Our results showed that 21 cases in GSE62080 could be classified into four of the SFM subtypes (Fig. 2a, Supplementary Fig. 2a, and Supplementary Table. 8). All of the SFM-E and SFM-F were responsive to FOLFIRI, and eight out of nine FOLFIRI responsive CRCs were defined as SFM-E and SFM-F $(P=0.0006)$. On the contrary, SFM-A and SFM-B were resistant to FOLFIRI. Similarly, we also tested another chemotherapy regimen FOLFOX response in combined 


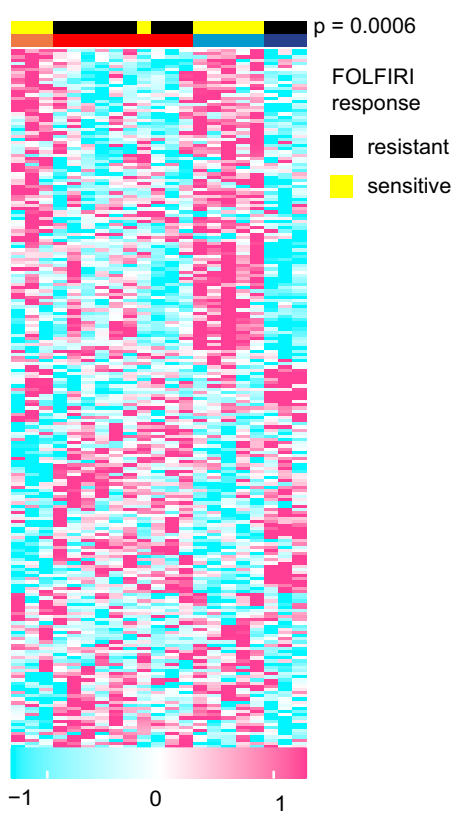

C

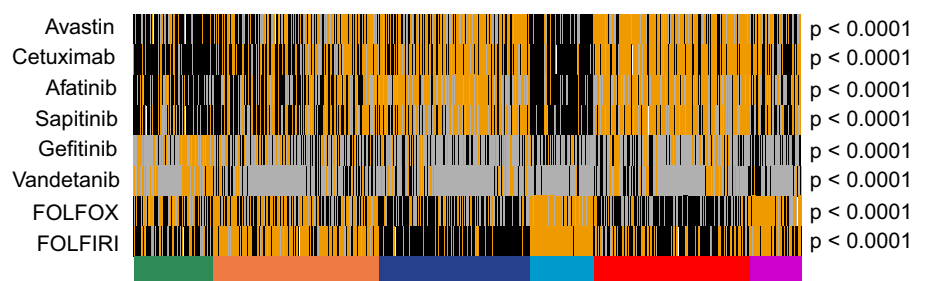

SFM subtype

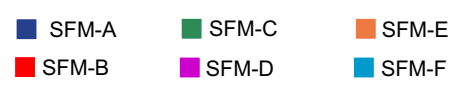

d
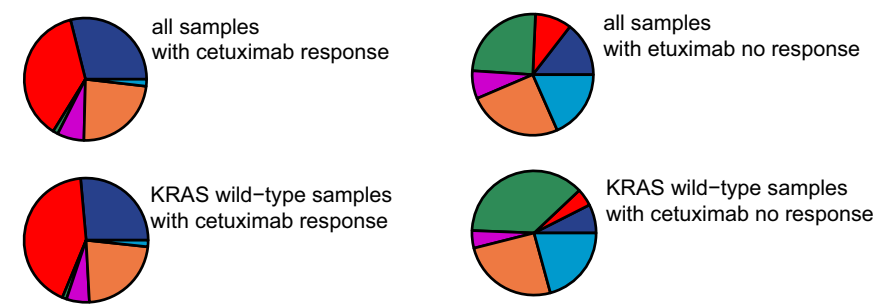

e

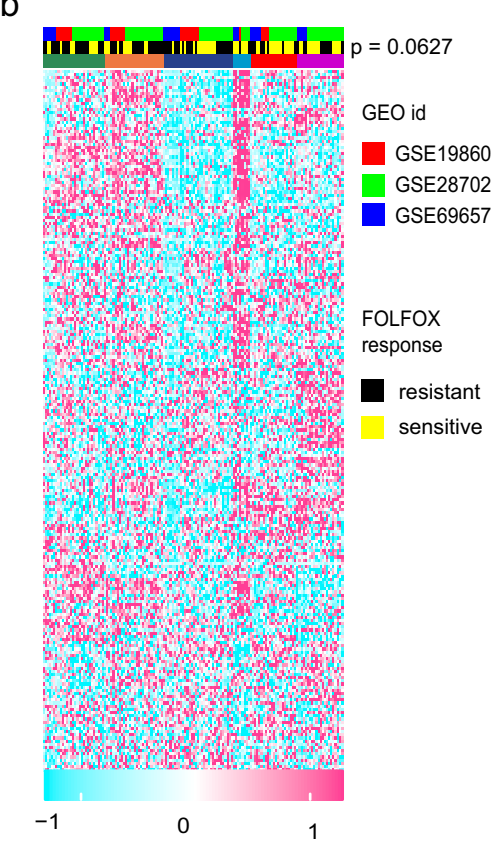

drug association

with response

with no response

undetermined (FDR $>0.2)$

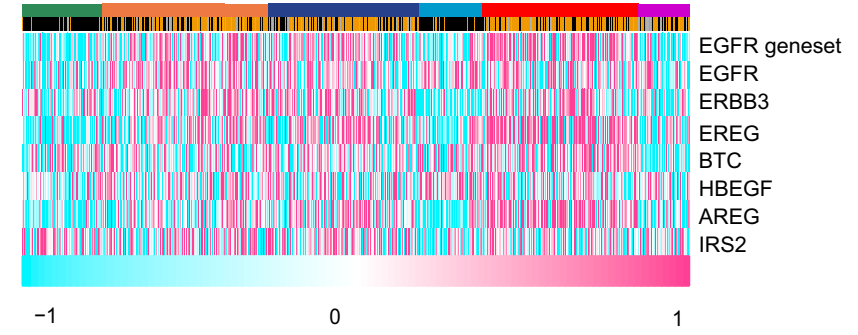

f
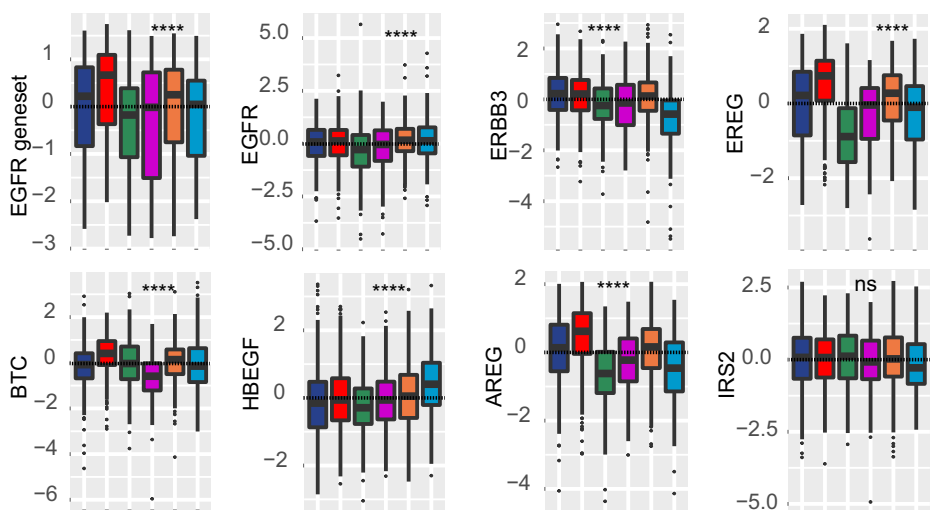
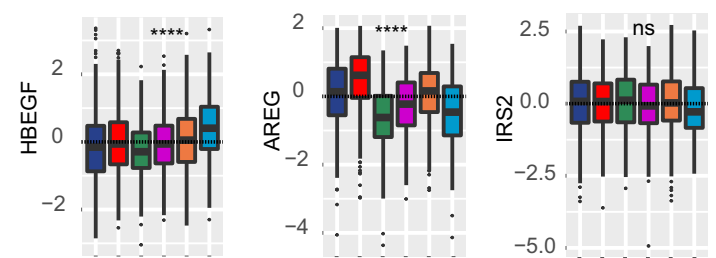

Fig. 2 Distinct sensitivity among SFM subtypes to FOLFIRI and FOLFOX chemotherapy regimens and RGFR inhibitors. a Heatmap showing individual response of patients to FOLFIRI and their association with subtypes in GSE62080 dataset $(n=21)$. $P$ value was calculated using Chisquared test. b Heatmap showing individual responses of patients to FOLFOX and their association with subtypes in combined three GEO datasets (GSE19860, GSE28702, and GSE69657, $n=142$ ). $P$ value was calculated using Chi-squared test. c Heatmap showing association of individual CRC patient's response to FOLFIRI, FOLFOX, and EGFR inhibitors. In these analyses, samples with FDR $<0.2$ were regarded as significant. d Prevalence of SFM subtypes regarded as sensitive or resistant to cetuximab in all CRC patients $(n=1752)$ or only in KRAS wildtype patients $(n=637)$. e Heatmap showing response to cetuximab, quantified expression of gene set of the EGFR pathway activity by applying GSVA and expression of individual genes of the EGFR gene set among SFM subtypes. $f$ Box plots of the EGFR gene set and individual genes of the EGFR gene set among SFM subtypes. Box hinges represent first and third quartiles, and middle represents the median. The upper and lower whiskers extend from hinges up and down indicate the most extreme values that are within $1.5 \times I Q R$ (interquartile range) of the respective hinge. 
datasets ( $n=142$, Fig. 2b, Supplementary Fig. 2b, and Supplementary Table. 8). Interestingly, we found that $75 \%$ of SFM-F ( $n=$ $6)$, as well as $36 \%$ of SFM-E $(n=10), 55 \%$ of SFM-D $(n=12)$ and $64 \%$ of SFM-B $(n=14)$ responded to FOLFOX $(P=0.0627)$. In addition, we included another two datasets (GSE72970 and GSE104645) in which samples were treated with FOLFIRI or FOLFOX, and the survival information was available. A total of 158 samples were classified into five main SFM subtypes (Supplementary Fig. 2c). Although there was no significant difference among the subtypes in terms of the response $(P=$ 0.1003 , Supplementary Fig. 2d) with more than half of SFM-A/B resistant, but $75 \%$ of SFM-C responsive to FOLFIRI or FOLFOX. Given that there were no significant differences in survival between responder and nonresponder samples in these two datasets, respectively, as indicated by $\mathrm{He}$ et al. ${ }^{28}$, we compared the survival differences among SFM subtypes. Interestingly, our results demonstrated that SFM-A/B had shorter OS and PFS, while SFM-C and $-E$ had better survival, and SFM-D and $-F$ were intermediate (log-rank $P<0.000$ for OS, $P<0.0001$ for PFS, Supplementary Fig. $2 e, f)$. Hence, these results to some extent suggested that at least SFM-A/B subtype could not benefit from FOLFIRI or FOLFOX treatment. To comprehensively compare the drug-response differences among SFM subtypes, we applied previous drug gene signatures to gene expression profiles using NTP algorithm, including FOLFIRI, FOLFOX, and vascular endothelial growth factor (VEGF) or epidermal growth factor receptor (EGFR) inhibitors (Supplementary Table. 5). Overall, the drug sensitivity among SFM subtypes were distinctive (Fig. 2c, Supplementary Fig. 2g, and Supplementary Table. 8). Specifically, the FOLFIRI response signature was significantly (false discovery rate, $F D R<0.2$ ) associated with $99 \%(n=166)$ of SFM-F, $80 \%(n=328)$ of SFM-E, and $67 \%(n=84)$ of SFM-D subtype samples, as compared to only $10 \%(n=37)$ of SFM-A, $30 \%(n=108)$ of SFM-B, and $28 \%(n=50)$ of SFM-C subtype $(P<0.0001)$. Similar results could also be found for FOLFOX response $(P<0.0001)$. We also applied another FOLFOX response signature of five gene pairs which has shown good performance to do the prediction by NTP or the way used in corresponding study ${ }^{28}$. Similar results were obtained in these two ways (Supplementary Fig. $2 \mathrm{~h}$, i), but not consistent with what we found that SFM-D/E/F were prone to response to FOLFOX. Further analysis on these two FOLFOX gene signatures, five gene pairs signature from $\mathrm{He}$ et al. $^{28}$ and 315 gene signature from Tong et al. ${ }^{29}$ suggested that there were no overlapped genes between these two gene signatures, implying the bias between them. Indeed, the $\mathbf{3 1 5}$ gene signatures were more robust because these genes were not only differentially expressed between responders and nonresponders in both pre-chemotherapy and postchemotherapy samples, respectively, but also validated between parental and resistant cancer cells. In addition, we found that most of the SFM-A and SFM-B were significantly (FDR $<0.2)$ correlated with EGFR inhibitors $(P<0.0001$, Fig. 2c). For instance, $68 \%(n=$ $237)$ of SFM-A, $80 \%(n=305)$ of SFM-B, and $50 \%(n=193)$ of SFM$E$ responded to cetuximab. Similar results could be observed for avastin, afatinib, and sapitinib. The results also indicated that SFM$C$ was responsive to EGFR tyrosine kinase inhibitors, including gefitinib and vandetanib. Ninety one percentage $(n=100)$ and $96 \%(n=96)$ of SFM-C were strikingly associated with gefitinib and vandetanib response signature, respectively. As one of the EGFR-specific monoclonal antibody, cetuximab has been applied to treatment for mCRC harboring KRAS wild type. However, some studies also implied that cetuximab did not have obvious benefit in chemotherapy regimens regardless of KRAS status ${ }^{30}$. Thus, we only included KRAS wild-type samples to further validate cetuximab sensitivity across SFM subtypes. Again, we found that SFM-A-B-E could predict the cetuximab response (Fig. 2d). This result was more notable when combining these three SFM subtypes compared with the remaining SFM subtypes (Supplementary Fig. 2j, k). In view of this, several genes involved with
EGFR pathway activity have been suggested to be associated with cetuximab response ${ }^{31-33}$. Consistently, this specific gene set showed higher expression in SFM-A-B-E subtypes (Fig. 2e, $f$ and Supplementary Fig. 2l). It is also overt after combining SFM-A-B-E compared with the rest irrespective of KRAS phenotype (Supplementary Fig. $2 \mathrm{~m}, \mathrm{n}$ ). To further confirm the association between EGFR inhibitors and SFM subtypes, we applied SFM signature to a combined dataset where samples were treated with cetuximab, GSE5851 (ref. ${ }^{32}$ ) and PRJEB34338 (ref. ${ }^{34}$; total $n=95$ ). Considering the small sample size, three main clusters were identified including SFM-A/B, SFM-C, and SFM-D/E/F (Supplementary Fig. 2o). Overall, nearly half of SFM-A/B samples were responsive to cetuximab, but lowest fraction for SFM-C $(20 \%, P=0.0421$, Supplementary Fig. 2p). Consistently, most of the EGFR pathwayassociated genes were highly expressed in SFM-A/B, but not for SFM-C (Supplementary Fig. 2q, r). Together, these findings suggest that SFM subtypes have predictive value of cetuximab response regardless of KRAS phenotype.

\section{Distinctive TME among SFM subtypes}

Since the SFM signature could discriminate TME, we further compared TME component among SFM subtypes. Firstly, we explored cell fractions among SFM subtypes by CIBERSORT. Based on this algorithm, we found that SFM subtypes displayed different enrichment for immune cell populations (Fig. 3a, Supplementary Fig. 3a-c, and Supplementary Table. 9). SFM-A was enriched with memory B cells, memory CD4 T cells, regulatory T cells (Tregs), plasma cells, and rested dendritic cells and rested NK cells. SFM-B was characterized by increased memory CD4 T cells, activated dendritic cells, rested NK cells. SFM-C displayed high infiltration of activated NK cells, follicular helper T cells, M1 macrophage, activated mast cells, and neutrophils. SFM-D exhibited enrichment with activated naive CD4 T and B cells, plasma cells, CD8 T cells, and Tregs. SFM-E showed increases of follicular helper T cells, MO/ 1 macrophages, and neutrophils. SFM-F was enriched with naive $B$ cells and macrophages, rested mast cells and neutrophils. In addition, SFM-D-E-F had displayed higher proportions of endothelial cells and fibroblasts (Fig. 3b, c). SFM-F had predominant stroma components with higher score of TGF- $\beta$ response (Fig. $3 d$, e). As for clinical features of immunotherapy, we found that SFM-C displayed highest neoantigen production follow by SFM-F (Fig. 3f, g). Consistently, SFM-C-F exhibited higher mutation burden than other subtypes (Fig. 3h, i). Based on several gene signatures, we found that SFM-F was also enriched with exhausted T cells and myeloid-derived suppressor cells (MDSCs; Fig. 3j, k). Importantly, we found that checkpoint biomarkers were highly expressed in SFM-C-F, including CD274, PDCD1, and CTLA4 (Fig. 3I-n). These suggested that SFM-C-F were $T$ cell suppressive. Further, both SFM-C and F were characterized as "hot" tumor and were responsive for IFN- $\gamma$ response (Fig. 3o, p). However, SFM-F was enriched with innate anti-PD1 resistance (IPRES) gene signature (Fig. $3 q, r$ ) and displayed higher IPRES score, which meant that SFM-F had features of "nonresponder" of immunotherapy. Results above indicated that even though SFM-C and $-\mathrm{F}$ were $\mathrm{T}$ cell suppressive, they responded differently to immunotherapy. This might be explained by that SFM-C was enriched with MSI phenotype, in which the immune suppressive could be blocked by immune inhibitors, while SFM-F was enriched with stroma/EMT phenotype that could also lead to immune suppressive, but not reversed by immune inhibitors.

\section{SFM subtype was an independent predictor of CRC}

We examined SFM subtypes with survival to test its prognostic value. We first performed prognostic analysis in each dataset independently regardless of treatment (chemotherapy or radiotherapy) or AJCC stage. Interestingly, we saw significant association between SFM subtypes and DFS or OS (Fig. 4a-d), which was 


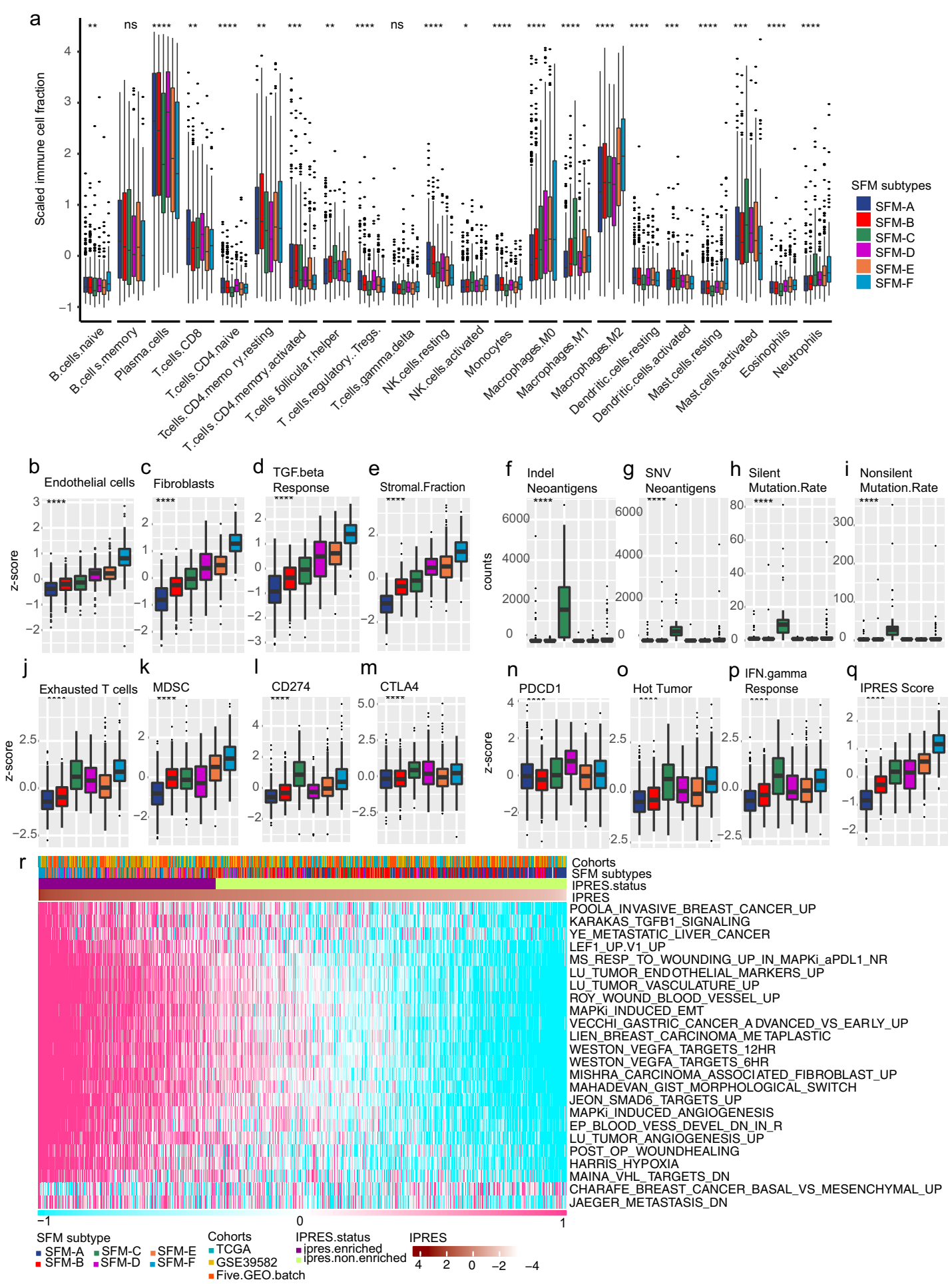

Fig. 3 Immune and TME-associated differences across SFM subtypes. a Estimate immune cells filtration using CIBERSORT in aggregated dataset. The fraction of immune cells were plotted using box plot across SFM subtypes. Box plots of fractions of endothelial cells (b) and fibroblasts (c) by MCP-counter algorithm. TGF- $\beta$ response score using GSVA (d), stromal fraction using ESTIMATE (e), neoantigen production $(\mathbf{f}, \mathbf{g})$, tumor mutation burden (h, i), proportion of exhausted cells (j), and MDSC (k) using GSVA, expression differences of checkpoint biomarkers, including CD274 (I), CTLA4 (m) and PDCD1 (n), hot tumor signature score (o), IFN- $\gamma$ response score (p), and IPRES score (q) using GSVA. $\mathbf{r}$ Estimate IPRES signature using GSVA. Cases were annotated as "IPRES enriched" when IPRES score were $>0.35$ unless were annotated as "IPRES non-enriched". The statistical difference was examined usings the Kruskal-Wallis test. (ns, $P>0.05 ;{ }^{*} P<=0.05 ; * * P<=0.01 ;{ }^{* * *} P<=$ $0.001 ;{ }^{* * * *} P<=0.0001$ ). For $\mathbf{a}-\mathbf{q}$, box hinges represent first and third quartiles, and middle represents the median. The upper and lower whiskers extend from hinges up and down indicate the most extreme values that are within $1.5 \times$ IQR (interquartile range) of the respective hinge. 
a

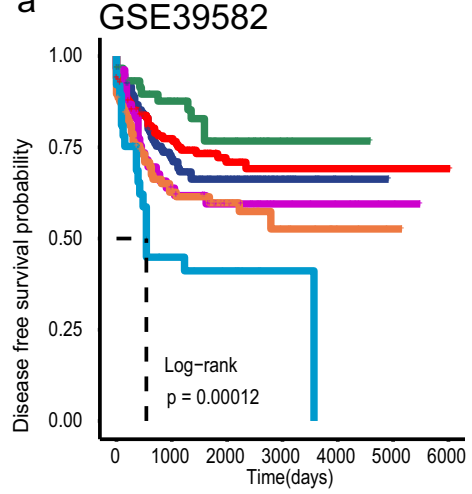

d

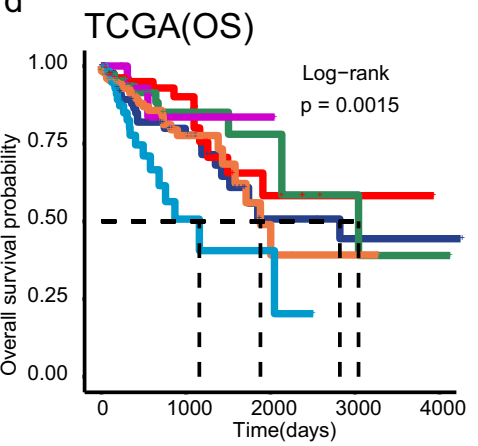

b

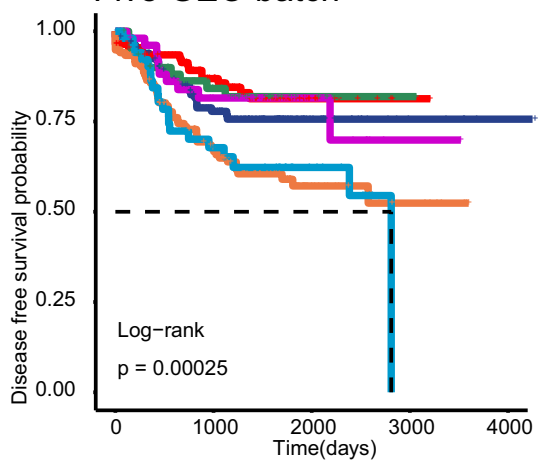

e samples with AJCC stage I-IV

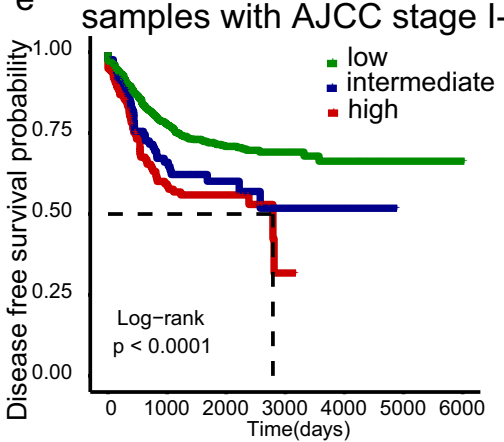

C TCGA(DFS)

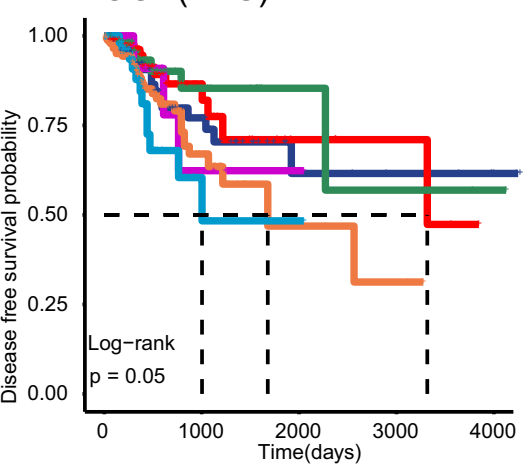

samples with AJCC stage II-III

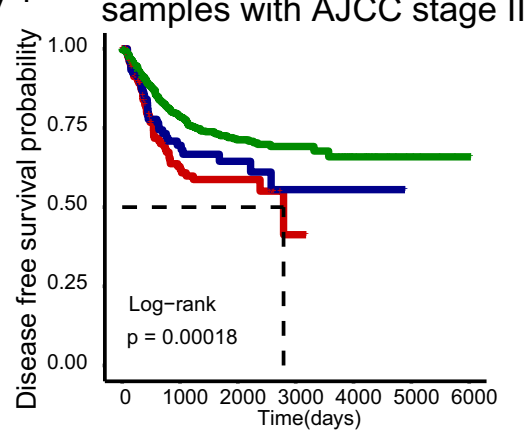

g samples with AJCC stage I-IV

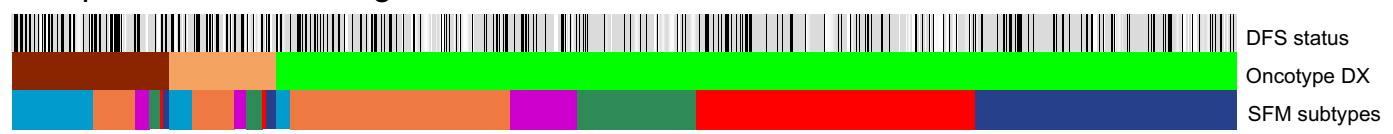

h samples with AJCC stage II-III
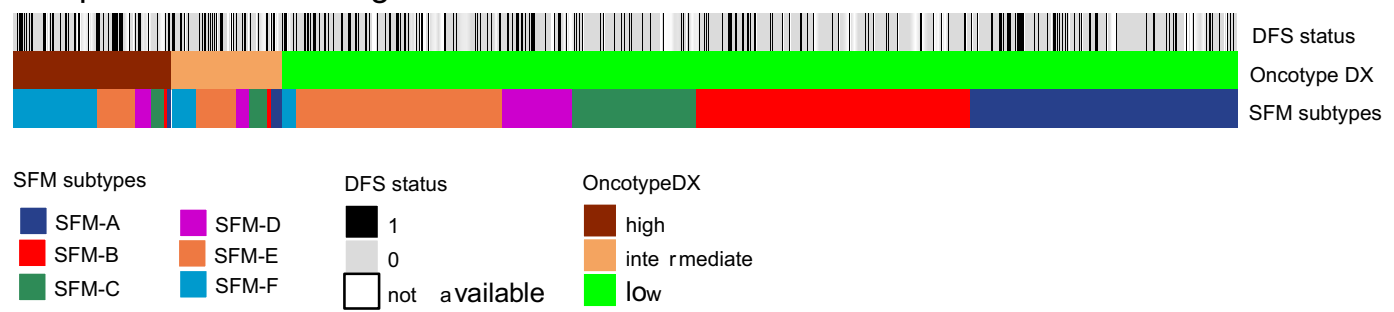

Fig. 4 Survival differences across SFM subtypes. Kaplan-Meier plots of DFS and OS in training dataset (a, GSE39582) and validation dataset (b, five GEO batch; c, TCGA for DFS; and d, TCGA for OS). e Kaplan-Meier plots of Oncotype DX risk groups in stage I-IV cases. f Kaplan-Meier plots of Oncotype DX risk groups in stage II-III cases. The tick marks on the Kaplan-Meier curves indicated the censored patients. The differences between the curves were determined by the log-rank test. $\mathbf{g}$ Comparison of SFM subtypes and Oncotype DX classification in stage I-IV cases. h Comparison of SFM subtypes and Oncotype DX classification in stage II and III cases.

more remarkable after three datasets were combined $(P<0.0001$, Supplementary Fig. 4a). Further, we assigned samples based on chemotherapy information. SFM subtypes still maintained significant association with DFS irrespective of chemotherapy (Supplementary Fig. 4b, c). The prognostic value of SFM subtypes were still significant in AJCC stage II and III patients, respectively, but not for stage I and IV patients probably because of the small sample size of stage I and IV patients (Supplementary Fig. $4 \mathrm{~d}-\mathrm{g}$ ). Altogether, this suggested that SFM-E and SFM-F had worse prognosis likewise. We combined SFM-E and SFM-F as a single high-risk group versus the remaining four to confirm this. The binary classifier displayed strong prognostic value as expected ( $P$ $<0.0001$, Supplementary Fig. $4 \mathrm{~h})$. In addition, we divided patents based on chemoradiotherapy information available. SFM subtypes showed significant prognostic value in non-chemoradiotherapy patients $(P<0.0001)$, but not in chemoradiotherapy patients $(P=$
0.11 , Supplementary Fig. $4 \mathrm{i}, \mathrm{j}$ ). Then we performed similar analysis using the binary classifier. Interestingly, high-risk group had worse prognosis in non-chemoradiotherapy cases $(P<0.0001)$, but not for chemoradiotherapy cases ( $P=0.095$; Supplementary Fig. $4 \mathrm{k}, \mathrm{I})$. Although the result was not significant might because of small number of samples with chemoradiotherapy information, we saw a trend that chemoradiotherapy could improve DFS in SFM-E and SFM-F subtypes. As the Oncotype DX recurrence score has been regarded as a prognostic classifier in colon cancer ${ }^{35,36}$, we evaluated its prognostic value within combined datasets. This score did have prognostic value in all AJCC stage cases $(P<$ $0.0001)$ or only stage II and III cases ( $P=0.00018$; Fig. $4 \mathrm{e}$, f). Then we compared the proportions of subtypes between SFM subtypes and Oncotype DX classifier. We found that $77 \%$ of high-risk cases and $75 \%$ of intermediate-risk cases identified by Oncotype DX classifier could be classified into SFM high-risk group (SFM-E-F; Fig. 
$4 g, h)$. This suggested that the SFM subtype had robust prognostic value. In addition, in univariate Cox regression analysis, most of the classifiers had at least one subtype that had significant difference (Supplementary Fig. 4m). However, when performed multivariate Cox regression analysis for each molecular subtype adjusted by age, gender, and AJCC stage, only SFM, CMS, CCS subtypes still had significant differences (Supplementary Fig. $4 \mathrm{n}$ ). CCS3 subtype $(P=0.0002, \mathrm{HR}=11.32,95 \% \mathrm{Cl}=3.14-40.84)$ had strongest prognostic value followed by SFM-F $(P=0.0001, \mathrm{HR}=$ 2.48, $95 \% \mathrm{Cl}=1.51-3.96)$ and SFM-E $(P=0.0031, \mathrm{HR}=1.89,95 \%$ $\mathrm{Cl}=1.24-2.89)$.

\section{Distinct gut microbiome patterns among SFM subtypes}

We performed the PathSeq algorithm in TCGA cohort. We acquired relative abundance value of 1093 microbes at the species level in 415 cases annotated with CRC subtypes and found that almost SFM subtypes harbored distinct bacterial communities (Fig. 5). Supplementary Table 10 displayed the top 15 highly enriched genera in each SFM subtype. The highest enriched bacterial species in SFM-A were Micrococcus luteus and Propionibacterium acnes. Bacterial species that highly enriched in SFM-A had lower enrichment in SFM-D, including Staphylococcus aureus, Pseudomonas mendocina, and Acinetobacter baumannii, etc. SFM-B was highly enriched with Escherichia coli. In addition, SFM-C had high enrichment for Bacteroides thetaiotaomicron, Fusobacterium nucleatum, and Bacteroides fragilis. SFM-D was enriched for Microbacterium testaceum, Rhodopseudomonas palustris, etc. And SFM-F displayed high enrichment for Corynebacterium aurimucosum and Pseudomonas putida. However, compared to other SFM subtypes, we did not see significantly enriched genera in SFM-E.

\section{DISCUSSION}

CRC is a disease with high heterogeneity just like other tumor types. The possible sources of heterogeneity of CRC derive from many aspects, such as genetic alterations, diversity of TME cell populations, and even the specific complex microbial community of gut. Increasing evidence indicated that TME has made great contribution to the development and progression of CRC. Gene expression profiles that can distinguish TME probably help to explain the heterogeneity of tumors. FOLFIRI and FOLFOX have been recommended as first-line backbone chemotherapy of $\mathrm{mCRC}$ by the Europe Society for Medical Oncology guidelines ${ }^{37}$. Although FOLFIRI or FOLFOX can significantly extend the median OS to $>15$ months, there are nearly $50 \%$ patients are not responsive ${ }^{38}$. Therefore, screening out those potentially responsive patients is also urgent. In this study, we took the advantages of comprehensive datasets to explore the heterogeneity of CRC, trying to identify CRC subtypes that are distinguished among TME and drug-response sensitivity, and helpfully, contributing to the precise treatment of CRC.

We firstly built a SFM gene signature that not only could discriminate TME, but also was associated with FOLFIRI resistance. Although the importance of TME has been addressed, none published gene signatures for CRC classification focused on either TME-associated genes or combination of TME and cytotoxic treatment. We found that SFM signature had limited overlap with previous gene signatures, while SFM subtypes displayed large overlaps with these gene signature-derived subtypes. The potential reason might be that the SFM signature were derived from (i) transcription of sorted cell populations which were more discriminative for TME than those signatures; and (ii) transcription associated FOLFIRI response. This indicated that SFM was unique and specific which encouraged us to test if SFM could help to identify CRC subtypes. The large overlaps between SFM subtypes and previous reported molecular subtypes might because that since these molecular subtypes were focused on CRC using the same datasets, the main potential subtypes based on transcription pattern would not change too much, such as stromal predominate subtype. Indeed, the SFM subtype was well-captured of the transcription signals derived from TME which made it possibly comparable with other subtypes even though it was limitedly overlapped with other gene signatures. Future investigation is needed to confirm the specific biological functions of SFM genes. We chose $K$-means to perform the classification because it is a straightforward clustering algorithm, which can compute faster than hierarchical clustering when there are numbers of variables. In addition, $K$-means is easy to produce tighter clusters than hierarchical clustering ${ }^{39}$. Finally, we identified six SFM-based subtypes with distinctive molecular and clinical relevance (Fig. 6).

Specifically, we identified the SFM-C subtype that was highly enriched for MSI tumors with BRAF mutation, CIMP+, hypermutation, and proximal colon phenotypes. This was in line with previous report about the association among these characteristics ${ }^{40}$. On the contrary, tumors classified as SFM-A-B-D-E showed $\mathrm{CIN}+$, MSS, and non-hypermutant features. We found mutation status of 53 CRC driver genes were significant different among SFM subtypes, which might reflect CRC intrinsic traits. Moreover, the association between previous existed gene signatures with SFM subtypes also uncovered underlying biological traits behind SFM subtypes. For instance, we observed significant association of serrated precursor neoplasia with SFM-C-E-F. This was consistent with previous report that sessile serrated polyps correlated with MSI tumors ${ }^{41}$. As SFM-E and SFM-F were not enriched for MSI tumors, we speculated that these two subtypes were associated with traditional serrated polyps ${ }^{41}$. Further pathological investigations about these correlations would be acquired. In terms of cell origins, our findings also indicated that SFM-B-E-F were derived from colon crypt base as these subtypes harbored hallmarks of colon crypt base cells. This means these subtypes might capture distinct colonic epithelial cell differentiation-associated pathways compared to other SFM subtypes. In terms of SFM-E and SFM-F, we saw significant enrichment for EMT and stem-like features. Our survival analysis indicated that these two subtypes had shorter DFS compared to the rest SFM subtypes. This was in line with previous investigations that stem/serrated/mesenchymal CRC subtype had poor prognosis ${ }^{16,17}$. In addition, large overlaps of high-risk cases between Oncotype DX and SFM subtypes also implied the prognostic value of SFM subtypes. To compare the prognostic value of previous CRC classifiers, we performed univariate and multivariate Cox regression analysis. Among these nine classifiers, including AJCC stage and SFM subtypes, seven of them had sub-classifiers that were significant in the univariate regression model. However, when adjusted by age, gender, and AJCC stage, only SFM, CMS, and CCS subtypes were still significant which meant that these three molecular classifiers had strong prognostic value in CRC.

The heterogeneity of CRC might also determine the sensitivity of chemotherapy. Sadanandam et al. have reported that stem-like subtype tumors responded to FOLFIRI ${ }^{14}$. This was consisted with our results that SFM-E and SFM-F were enriched for stem-like tumors and responsive to FOLFIRI. Given that we have shown that SFM-E and $-F$ generally had shorter survival than the remaining subtypes, our analysis on combined datasets (GSE104645 and GSE72970) indicated that SFM-A/B had worse survival after treated with FOLFIRI or FOLFOX regimens, while SFM-D/E/F have improved their survival. This means that FOLFIRI or FOLFOX might be more suitable for SFM-D, -E, and $-F$ subtypes. Moreover, although SFM-C displayed nonresponsive to FOLFIRI and FOLFOX, which was also consistent with previous studies suggesting MSI tumors barely responded to fluorouracil-based therapy ${ }^{42}$, it had better survival in this combined dataset probably because of the enrichment of MSI phenotype, which has been proofed to have relative better survival ${ }^{43}$. Regardless of SFM subtypes, the response rate of FOLFIRI and FOLFOX were $48 \%$ and $47 \%$, 


\begin{tabular}{|c|c|c|c|c|c|c|c|c|}
\hline \multirow{2}{*}{\multicolumn{9}{|c|}{$P$ value }} \\
\hline & & & & & & & & \\
\hline $2.52 \mathrm{E}-08$ & -0.98 & 1.81 & -0.06 & -0.32 & 0.30 & -0.75 & Escherichia.coli & 1.5 \\
\hline 0.041 & -0.97 & 0.83 & -0.10 & 1.43 & -0.05 & -1.15 & Shigella.sonnei & 1 \\
\hline 0.128 & 0.16 & -1.20 & -0.93 & 1.59 & 0.01 & 0.37 & Paracoccus.denitrificans & 0.5 \\
\hline $6.62 \mathrm{E}-05$ & -0.77 & -0.81 & -0.11 & 1.91 & -0.27 & 0.05 & Oscillibacter.valericigenes & 0 \\
\hline 8.37E-08 & -0.17 & -0.53 & -0.51 & 2.02 & -0.37 & -0.45 & Rhodopseudomonas.palustris & -0.5 \\
\hline $5.41 \mathrm{E}-04$ & -0.21 & -0.66 & -0.60 & 1.99 & -0.11 & -0.42 & Microbacterium.testaceum & -1 \\
\hline 2.23E-04 & -1.06 & -1.24 & 0.51 & 0.95 & -0.21 & 1.05 & [Eubacterium].rectale & 5 \\
\hline 4.64E-04 & -1.19 & -1.08 & 0.90 & 0.56 & -0.29 & 1.10 & Roseburia.hominis & \\
\hline $1.31 \mathrm{E}-04$ & -1.15 & -1.02 & 0.54 & 1.41 & -0.31 & 0.52 & [Clostridium].saccharolyticum & \\
\hline 0.302 & -1.30 & -0.18 & 0.02 & 1.57 & -0.69 & 0.58 & Bacteroides.vulgatus & \\
\hline 0.058 & 1.52 & 1.03 & -0.74 & -0.54 & -0.60 & -0.67 & Streptococcus.pneumoniae & \\
\hline 0.094 & 1.39 & -0.04 & 0.19 & -1.71 & -0.11 & 0.29 & Acinetobacter.baumannii & \\
\hline 4.59E-03 & 1.63 & 0.01 & -0.52 & -1.42 & 0.20 & 0.10 & Staphylococcus.epidermidis & \\
\hline $1.55 \mathrm{E}-08$ & 1.66 & -0.61 & -0.23 & -1.26 & -0.05 & 0.49 & Pseudomonas.mendocina & \\
\hline $1.67 \mathrm{E}-07$ & 1.45 & -0.38 & -0.13 & -1.53 & -0.03 & 0.62 & Agrobacterium.sp..h13-3 & \\
\hline 0.026 & 1.71 & -0.38 & -0.75 & -0.92 & 0.65 & -0.32 & Staphylococcus.aureus & \\
\hline 4.52E-03 & 1.99 & -0.25 & -0.64 & -0.70 & -0.23 & -0.17 & Micrococcus.luteus & \\
\hline 0.039 & 1.81 & -0.78 & -0.95 & -0.27 & -0.17 & 0.36 & Propionibacterium.acnes & \\
\hline $2.12 \mathrm{E}-04$ & -0.42 & -1.21 & 1.54 & 0.53 & -0.77 & 0.34 & Bacteroides.fragilis & \\
\hline $1.71 \mathrm{E}-05$ & -0.38 & -0.88 & 1.82 & 0.07 & -0.84 & 0.22 & Fusobacterium.nucleatum & \\
\hline 0.051 & -0.28 & -0.94 & 1.91 & -0.20 & -0.59 & 0.10 & Bacteroides.thetaiotaomicron & \\
\hline 0.057 & 0.37 & -0.92 & -0.46 & -0.15 & -0.67 & 1.83 & Corynebacterium.aurimucosum & \\
\hline 0.091 & -0.16 & -1.45 & -0.29 & 0.20 & 0.04 & 1.66 & Pseudomonas.putida & \\
\hline 0.223 & 0.39 & -1.25 & 1.24 & -1.07 & -0.10 & 0.79 & Pseudomonas.stutzeri & \\
\hline $6.76 \mathrm{E}-04$ & 0.61 & -0.58 & 0.11 & -1.67 & 0.37 & 1.16 & Ralstonia.pickettii & \\
\hline $1.84 \mathrm{E}-03$ & 0.46 & -1.22 & 0.09 & -1.05 & 0.27 & 1.45 & Pseudomonas.fluorescens & \\
\hline 0.019 & 0.80 & -1.23 & 0.28 & -1.06 & -0.07 & 1.28 & Pseudomonas.aeruginosa & \\
\hline
\end{tabular}

Fig. 5 Heatmap showing the relative abundance of dominant bacterial across SFM subtypes. Kruskal-Wallis test was used to test the differences across SFM subtypes.

respectively in the aggregated datasets $(F D R<0.2)$. This was consistent with the previous report that nearly half of CRC were responsive to these two treatments ${ }^{38}$. In addition, SFM-A and SFM$B$ displayed high sensitivity of VEGF/EGFR inhibitors. Importantly, as for cetuximab, our results suggested that these subtypes were enriched for overexpression of ERBB3, EREG, BTC, and AREG. These biomarkers have been proved to be involved in EGFR-associated pathways blockade ${ }^{31-33}$. Although the sensitivity of most samples for gefitinib and vandetanib could not be accurately evaluated $($ FDR $<0.2)$ across SFM subtypes, we saw SFM-C subtype was responsive to these two inhibitors. FOLFIRI or FOLFOX have been approved to be used as the standard chemotherapy treatments plus avastin or cetuximab, while our results indicated that not all CRCs are suitable for these combined regimens.

Moreover, increasing evidence suggested that MSI tumors were responsive to immunotherapy of anti-PD1/PDL1 ${ }^{9,10}$. SFM-C displayed higher mutation burden with MSI phenotype. Our results also indicated that SFM-C and SFM-F were immune suppressive as these two subtypes had higher proportions of exhausted $T$ cells and MDSCs and IFN-yresponse rate. Both of them were highly expressed of CD274 and CTLA4. Since SFM-F exhibited higher IPRES score, which meant that SFM-F had features of "nonresponder" of immunotherapy. These results indicated that both of MSI and high stroma/EMT microenvironment could lead to immune suppression, while MSI-induced immune suppression could easily be blocked by checkpoint inhibitors, but not for stroma/EMT-induced immune suppression. This might be reason why part of patients with high checkpoint markers did not response to checkpoint inhibitors.

So far, the association of CRC molecular subtypes with gut microbiome has not been clearly elucidated. The first time to describe the association of bacterial signatures with CRC molecular subtypes was reported by Burns et al. ${ }^{44}$. We found distinctive bacterial communities across SFM subtypes by mapping TCGA CRC nonhuman RNA-sequencing reads to bacterial reference sequences. Notably, SFM-C with MSI tumor showed high relative abundance of $F$. nucleatum, which has been proved to be associated with CRC development and progression ${ }^{45,46}$. F. nucleatum also has strong association with immune response of CRC, particularly by recruiting $T$ cells. Our results were also in line with previous reports that $F$. nucleatum was associated with CIMP+, MSI CRC subtype ${ }^{47}$, both of which were features of SFM-C. E. coli was significantly enriched in SFM-B. E. coli has been regarded as commensal bacterial in gut microbiota, but some of its stains might also contribute to tumor development by inducing chronic inflammation or producing toxins ${ }^{48}$. P. acnes, enriched for SFM-A has shown association with prostate cancer ${ }^{49}$. $P$. acnes can induce prostatic inflammation in prostate cancer glands. As the biological functions of these distinctive gut microbes have not been clearly elucidated, the gap between these gut microbes and CRC should be bridged by performing more experimental studies and clinical trials.

The limitations should be acknowledged for this research. Firstly, this study is retrospective, the SFM subtypes classification should be further identified in large prospective clinical trials. Secondly, when performing multivariate Cox regression analysis, not all of the clinical factors were included, such as tumor grade and number of positive nodes as these information was not publicly available. Thus, SFM subtypes as independent prognostic factor needs to be further confirmed. Thirdly, we predicted drug response using previous gene signatures, since these information are not available in the included datasets. Therefore, drug sensitivity among SFM subtypes should be confirmed using more comprehensive data in the future. Fourthly, the SFM gene signature was initially correlated with FOLFIRI response, while 


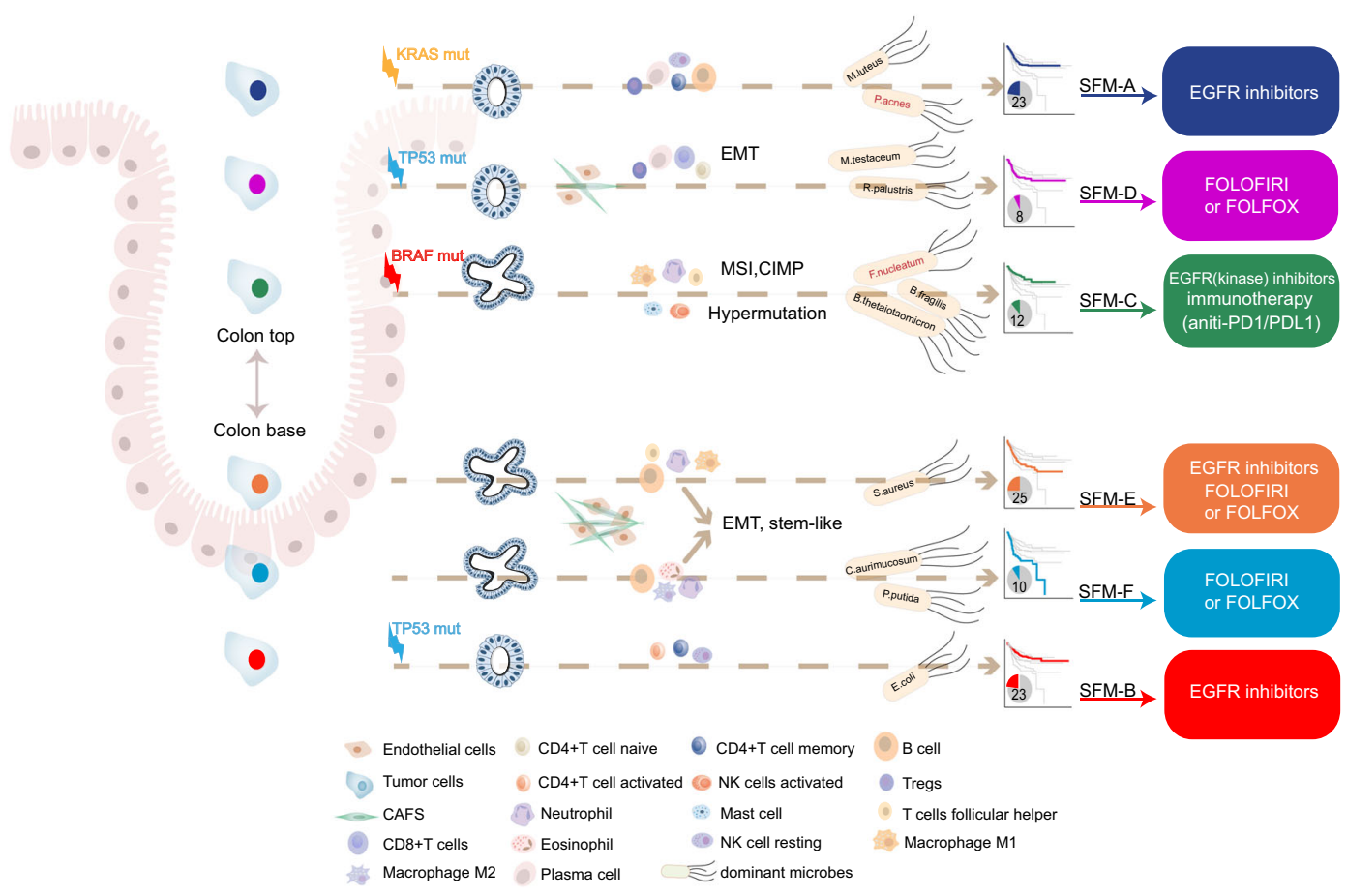

Fig. 6 Summary of characteristics of SFM subtypes. These included pathological features, genomic markers, TME, epigenetic changes, gut microbes, prognostic value, and possible SFM subtypes-guided therapies for CRC.

we saw SFM subtypes can also predict EGFR inhibitors response, which could not be well interpreted based on our current analysis.

In conclusion, we build a new classifier of CRC into six molecular subtypes, using SFM signature that can discriminate TME and is associated with drug response. This gene signature can partially explain the heterogeneity of CRC. The SFM subtypes would help to improve precision treatment of CRC.

\section{METHODS}

\section{CRC data collection and candidate gene selection}

The publicly available datasets used in this study were accessed from the Gene Expression Omnibus (GEO) and TCGA. Among these datasets, three datasets (GSE39395, GSE39396, and GSE62080) were used for SFM signature construction. Four datasets were used for CRC classification using SFM, including GSE39582, five GEO batch, TCGA, and Renji cohort. Five GEO batch consisted of five GEO datasets, including GSE14333, GSE17536, GSE17537, GSE33113, and GSE37892. Four scRNA-seq data were used for SFM validation, including GSE81861 for CRC, GSE103322 for HNSCC, GSE72056 for melanoma, and GSE75688 for BRCA. In addition, some aggregated datasets were used for drug-response exploration, including (i) combined GSE19860, GSE28702, and GSE69657 for FOLFOX response; (ii) GSE104645 and GSE72970 for FOLFOX or FOLFIRI response; and (iii) GSE5851 and PRJEB34338 for cetuximab response.

For GEO datasets, we first performed the robust multi-array average method within "affy" $R$ package to normalize each dataset ${ }^{50}$. Three datasets named GSE39395, GSE39396, and GSE62080 were then used to do differential expression analysis to construct a SFM signature, in which genes could discriminate TME and were associated FOLFIRI sensitivity. In GSE39395 and GSE39396 (ref. ${ }^{51}$ ), FACS was used to separate cell subpopulations from eight and six samples, respectively $\left(C D 45^{+}\right.$Epcam ${ }^{-}$ for immune cells, CD45 ${ }^{-}$Epcam $^{+}$for tumor epithelial cells and $\mathrm{CD} 45^{-} \mathrm{Ep}-$ $\mathrm{cam}^{-}$for stomal cells in GSE39395; $\mathrm{CD}^{+} 5^{+} \mathrm{EPCAM}^{-} \mathrm{CD} 31^{-} \mathrm{FAP}^{-}$for immune cells, $\mathrm{CD}_{4} 5^{-} \mathrm{EPCAM}^{+} \mathrm{CD} 31^{-} \mathrm{FAP}^{-}$tumor epithelial cells, $\mathrm{CD} 45^{-} \mathrm{EP}-$ $\mathrm{CAM}^{-} \mathrm{CD} 31^{+} \mathrm{FAP}^{-}$endothelial cells, and $\mathrm{CD}^{-} 5^{-} \mathrm{EPCAM}^{-} \mathrm{CD} 31^{-} \mathrm{FAP}^{+}$for CAFs in GSE39396). GSE62080 contained transcriptomic data from nine FOLFIRI responders and 12 nonresponders ${ }^{52}$. We did differential expression analysis using limma $\mathrm{R}$ package ${ }^{53}$ between each two of the cell populations in GSE39395 and GSE39396, respectively. For instance, in GSE39395, there were three cell populations, including immune cells, tumor epithelial cells, and stomal cells, we thus did differential expression analysis for three times, including immune cells versus tumor epithelial cells, immune cells versus stromal cells, and tumor epithelial cells versus stromal cells. Similar strategies were applied to GSE39396. As for GSE62080, differential expression analysis was directly performed between FOLFIRI responders and nonresponders. Each time for differential expression analysis, candidate genes were identified as differentially expressed when $P$ value was $<0.05$ and $|\log \mathrm{FC}| \geq 1$. Further, we selected out differentially expressed genes within each of these three datasets. Finally, differential expressed genes among these three datasets were overlapped, and further termed as SFM gene signature, as shown in Fig. 1a.

GSE39582 consisted of 566 CRC samples was used as a discovery dataset $^{23}$. To construct a large dataset for validation, we combined five GEO datasets as a unit (GSE14333, GSE17536, GSE17537, GSE33113, and GSE37892), referred to "five GEO batch" dataset ${ }^{54-57}$. Samples that overlapped between GSE14333 and GSE17536 were excluded from GSE14333. The ComBat method within "sva" R package was used for batch correction ${ }^{58}$. By doing so, the first validation dataset (five GEO batch) were composed of 609 CRC cases. In addition, we directly downloaded 577 TCGA CRC gene expression profiles from the synapse repository of CRC provided by the CRCSC ${ }^{15}$. This level 3 TCGA data was used as the second validation dataset. As for drug-associated datasets, we selected three GEO datasets where samples were treated with FOLFOX: GSE19860, GSE28702, and GSE69657, and combined these three datasets together after batch correction $^{59,60}$. This constructed a gene expression profile derived from 78 nonresponders and 64 responders of FOLFOX. In addition, another two datasets in which samples were treated with FOLFIRI or FOLFOX: GSE104645 and GSE72970 were also combined after batch correction ${ }^{61,62}$. Finally, two datasets, GSE5851 and PRJEB34338 where samples were treated with EGFR inhibitor, cetuximab, were also combined after batch correction $^{32,34}$. We also downloaded four scRNA-seq datasets for validation, including GSE81861 for CRC ${ }^{63}$, GSE103322 for HNSCC ${ }^{64}$, GSE72056 for melanoma $^{65}$, and GSE75688 for BRCA ${ }^{66}$. The annotated cell types information are available from corresponding original papers. To account for the influences of technical noise, we firstly performed missing data imputation and data normalization to gene expression profiles. ScImpute algorithm was used to impute missing gene expression with default parameters and TPM, or raw counts value and gene lengths ${ }^{67}$. We used "scater" R packages to normalize imputed raw counts ${ }^{68}$. As for HNSCC and melanoma datasets which had far enough cells, tumors, and nonmalignant cells types containing $<50$ cells were excluded for further analysis. 
Another total of 53 CRC samples were collected from Renji hospital. The study protocol was approved by the ethics committee of Shanghai Jiao Tong University School of Medicine. Written informed consent was obtained from all patients. All samples were sequenced on an Illumina HiSeq 4000 for $2 \times 150$-bp paired-end sequencing. Reads were mapped to the human genome (GRCh38) using HISAT2 v2.10 (https://ccb.jhu.edu/ software/hisat2/), with the default options ${ }^{69}$. Count files of the aligned sequencing reads were generated by the featurecount using the Gencode version $22 \mathrm{gtf}$ file (https://www.gencodegenes.org/human/) ${ }^{70}$. The read counts from each sequenced sample were combined into a count file, which was subsequently used for the downstream expression analysis.

Clinical data were directly downloaded from corresponding GEO website or supplementary materials from associated literatures. Clinical information for TCGA CRCs was downloaded from CRCSC in synapse database and immune associated features were downloaded from a recently public research ${ }^{71}$.

\section{Identification of SFM subtypes using $K$-means clustering algorithm}

Based on the SFM, the identification of SFM subtypes was first performed in discovery dataset (GSE39582) by applying $K$-means clustering algorithm implemented in "factoextra" R package.

We identified the optimal number of clusters by gap statistics within the predetermined number of clusters $(k)$ varying from 3 to 8 . Among these clusters, $k=6$ was selected with the best statistic in the discovery dataset. Then we evaluated the similarity and expression differences among the SFM subtypes with the cluster dendrogram and heatmap of the SFM, respectively. To validate the robustness of SFM subtypes, we further performed the same analysis in validation datasets (five GEO batch, TCGA, and Renji cohort).

We used $K$-means clustering algorithm to do the clustering since it is one of the simple and important clustering approach and statistically deterministic without specifying initial centers ${ }^{72,73}$. It is an easier way to classify dataset assuming $k$ clusters. One of the advantages of $K$-means algorithm is its higher computational speed for large variable when the number of clusters is relative small. We applied $K$-means clustering implemented in "factoextra" R package to gene expression profiles based on SFM signature comprised of 250 unique genes. Several aspects were considered to determine to cluster assignment in each dataset: (i) gap statistics were reported for each cluster which compared the total within intra-cluster variation for different values of $k$ with their expected values under null reference distribution of the data ${ }^{74}$. The estimate of the optimal clusters will be the value that maximize the gap statistics which means that the clustering structure is far away from the random uniform distribution of points. Given that $K$-means clustering requires to pre-specify the number of clusters, we set number of clusters varying from $k=3$ to 8 . Generally, the output of clustering can be visualized using "fvix_gap_stat" function in "factoextra" $R$ package which can suggest the optimal number of clusters marked as vertical dashed line. (ii) For a dataset that the optimal number of clusters were not given by the function itself among $k=3$ to $k$ $=8$, we visualized the dendrogram of the clustering and drew the heatmap showing the expression of SFM signature to facilitate the selection of number of clusters. We further selected out the number of clusters that (1) the height of dendrogram were good enough to discriminate amongst clusters as indicated by a red horizontal dashed line; (2) the gene expression profiles in the heatmap showed part of SFM genes that were discriminative amongst clusters, which might be subjective at this stage; and (3) additionally, as for a dataset with small number of sample size, we generally selected smaller number of clusters that also satisfied the principle above.

\section{Enriched functions and pathways of SFM subtypes}

To find to dysregulated signaling pathways among the SFM subtypes, we first did differential expression analysis in each SFM subtype versus the rest in discovery cohort and selected 2000 top up- and down-expressed genes for further analysis in each SFM subtype ${ }^{23}$. These genes were then applied in ClueGO and CluePedia apps ${ }^{75}$. These two plug-ins of Cytoscape are open-source Java tools that can extract the non-redendant biological information for a set of genes. In this study, we performed Ontology/ pathway analysis, including Gene Oncology (GO, BP, CC, MF, and immune system process) and Kyoto Encyclopedia of Genes and Genomes (KEGG) in Cytoscape 3.5.0 software.

\section{NTP implementation and signature adaptation}

NTP-based classification ${ }^{25}$ was performed on GenePattern (https://www. genepattern.org/). NTP classification allows us to apply given signatures to individual cases wherever these gene signatures are derived from. Generally, these gene signatures consist of upregulated and downregulated genes to form a binary reference gene expression. NTP applies a nearest neighbor method to calculate the similarity of gene expression profile to a reference gene expression signature in each case. Then a null distribution of similarity coefficients would be assessed by randomly subsampling the gene space. Finally, a $P$ value would be calculated when comparing the similarity coefficient derived from the given gene signature with the null distribution. The threshold selected for significance of each case was Benjamini-Hochberg-corrected FDR $<0.2$ (ref. ${ }^{14}$ ).

We evaluated the association of SFM subtypes with a set of gene signatures (Supplementary Table 5). The lists of gene signatures derived from previously published papers are as following: intestinal stem cell signature $^{76}$, colon crypt signature ${ }^{77}$, serrated CRC signature ${ }^{27}$, EMT signature $^{78}$, FOLFIRI response signature ${ }^{79}$, and FOLFOX $^{29}$ response signature and VEFG/EGFR inhibitors signatures described by Schutte et al. ${ }^{80}$, including avastin, cetuximab, afatinib, sapitinib, gefitinib, and vandetanib.

\section{Cells infiltration estimation}

We used CIBERSORT algorithm to estimate the immune cell infiltration in CRCs samples. This method used cell-specific gene signatures to discriminate a total of 22 immune cell populations as described by Newman et al. ${ }^{81}$. We additionally used microenvironment cell population (MCP)-counter algorithm to estimate the proportions of stroma and endothelial cells. This method can robustly quantify the abundance of various immune and stromal cell populations based on transcriptomic data for each sample ${ }^{82}$. The output of MCP-counter can be used to estimate the relative infiltration of endothelial cells, fibroblasts, and another eight immune cells populations. We performed MCP-counter analysis using "MCPcounter" R package. Stromal fraction was estimated using "estimate" $\mathrm{R}$ package.

\section{Survival analysis}

DFS and OS were regarded as the end points upon the clinical information available in the datasets (RFS in GSE39582 and five GEO batch; RFS/OS in TCGA). Survival analysis was performed based on the Kaplan-Meier algorithm. The $P$ value for the differences between SFM subtypes was calculated using log-rank test. Univariate and multivariate Cox models were constructed by cox proportion hazards regression. These analyses were implemented in "survival" and "survminer" R packages.

\section{Single sample gene set enrichment analysis}

Gene set variation analysis (GSVA) is a nonparametric and unsupervised method that can be used to evaluate gene set enrichment based on gene expression profiles derived from microarrays or RNA-seq data ${ }^{83}$. GSVA can evaluate the given pathway activity variation by transforming the gene by sample matrix into a gene set by sample matrix. Therefore, it can easily assess a pathway enrichment for individual case. Importantly, the GSVA also provide a method called "single sample gene set enrichment analysis (ssGSEA)", which can compute a gene set enrichment score per sample as the normalized difference in empirical cumulative distribution functions of gene expression ranks inside and outside a given gene set. Single sample gene set enrichment analysis (sSGSEA) was firstly described by Berbie et al. $^{84}$. In this study, we performed ssGSEA implemented in "GSVA" $R$ package and evaluated the EGFR gene set activity across the SFM subtypes in three datasets (GSE39582, five GEO batch and TCGA and Renji cohort). The EGFR gene set consisted of EGFR pathways-associated ligands or receptors, including EGFR, ERBB3, EREG, BTC, HBEGF, AREG, and IRS2 as previous papers reported ${ }^{31-33}$. Besides, we also performed similar analysis for TGF-beta response ${ }^{85}$, exhausted T cells ${ }^{86}$, MDSCs $^{86}$, hot tumor ${ }^{87}$, IFNgamma response $^{88}$, IPRES signatures ${ }^{89}$, and SFM gene signature in four single-cell datasets.

\section{Oncotype DX}

The 12-mRNA-based Oncotype DX colon cancer recurrence score assay was built based on transcriptomic data from 1851 cases with stage II and III colon cancer ${ }^{90}$. It has been recognized as an independent prognostic factor in CRC. To confirm the prognostic value of SFM subtypes, we 
proposed to associate the SFM subtypes, Oncotype DX with DFS in univariate and multivariate Cox regression models. To this end, we first reproduced the Oncotype DX calculation in three datasets as described by Clark-Langone et al. $^{36}$. Cases with recurrence score (RS) $<30,30 \leq$ $\mathrm{RS} \leq 41$, or RS $>40$ were regarded as low, intermediate or high risk of recurrence, respectively. The association of Oncotype DX with DFS was confirmed in all cases $(P<0.0001$, Fig. 4 e) or only stage II and III cases $(P$ $=0.00018$, Fig. 4f).

\section{Microbial detection using PathSeq algorithm}

The PathSeq algorithm described by Kostic et al. can be used to identify microbes according to deep sequencing data from RNA sequencing and WGS in human tissue ${ }^{91,92}$. The human reads would be computationally subtracted by mapping reads to human genome database after lowquality, duplicate, and repetitive sequences were filtered. Then mapped reads would be removed and unmapped reads that belong to nonhuman, pathogen-derived reads would be subjected to further analysis. Followed by the assignment of the unmapped reads to the acknowledged sequenced whole bacterial reference genomes by a metagenomic analysis, these unmapped reads would be taxonomically classified into bacterial, viral, and fungal sequences. The relative abundance value for each organism would be then computed using the reads mapping with $>90 \%$ sequence identity and $>90 \%$ query coverage. Finally, the classification was analyzed at the domain, phylum, genus, and species level. Following PathSeq approach, we obtained the relative abundance of 1093 microbes in 429 CRC samples, 415 of which were annotated with SFM subtypes information and analyzed afterwards.

\section{Statistical analysis}

We performed two-tailed Students's $t$ test, Fisher's exact test, $X^{2}$ test, and Kruskal-Wallis test using $\mathrm{R}$ program (v.3.4.1). Cox regression hazard model and Kaplan-Meier analyses were conducted using "survival" and "survminer" R packages, respectively. In all these tests, statistical significance was set at 0.05 . In the NTP algorithm, the results were regarded as significant if the Benjamini-Hochberg FDR was $<0.2$.

\section{Declarations}

\section{DATA AVAILABILITY}

The data generated and analyzed during this study are described in the following data record: https://doi.org/10.6084/m9.figshare. $13027715^{93}$. The data analyzed during the study were downloaded from public databases, including Gene Expression Omnibus (GEO; https://www.ncbi.nlm.nih.gov/geo/) and The Cancer Genome Atlas (TCGA; TCGA CRC datasets available from the Synapse repository at: https://www.synapse.org/\#!Synapse:syn2623706/files/). For a list of accession IDs for the analyzed data, see Supplementary Table S1. The Renji RNA-seq data is available from GEO: https://identifiers.org/geo:GSE158559 ${ }^{94}$. All other output data are included in the figshare data record ${ }^{93}$.

\section{CODE AVAILABILITY}

All statistical analyses described in the manuscript were performed using custom software developed in R. Custom code for the analysis is available in at https://github. com/helianthuszhu/SFM.subtype.CRC.

Received: 10 February 2020; Accepted: 21 October 2020; Published online: 12 February 2021

\section{REFERENCES}

1. Siegel, R. L., Miller, K. D. \& Jemal, A. Cancer statistics, 2018. CA Cancer J. Clin. 68, 7-30 (2018)

2. Molinari, C. et al. Heterogeneity in colorectal cancer: a challenge for personalized medicine? Int. J. Mol. Sci. 19, 3733 (2018).

3. Chen, F. et al. New horizons in tumor microenvironment biology: challenges and opportunities. BMC Med. 13, 45 (2015).

4. Whiteside, T. L. The tumor microenvironment and its role in promoting tumor growth. Oncogene 27, 5904-5912 (2008).

5. Fridman, W. H., Pages, F., Sautes-Fridman, C. \& Galon, J. The immune contexture in human tumours: impact on clinical outcome. Nat. Rev. Cancer 12, 298-306 (2012).
6. Andre, F. \& Pusztai, L. Molecular classification of breast cancer: implications for selection of adjuvant chemotherapy. Nat. Clin. Pract. Oncol. 3, 621-632 (2006).

7. Taube, J. M. et al. Association of PD-1, PD-1 ligands, and other features of the tumor immune microenvironment with response to anti-PD-1 therapy. Clin. Cancer Res. 20, 5064-5074 (2014).

8. Mlecnik, B. et al. Integrative analyses of colorectal cancer show immunoscore is a stronger predictor of patient survival than microsatellite instability. Immunity 44, 698-711 (2016).

9. Xiao, Y. \& Freeman, G. J. The microsatellite instable subset of colorectal cancer is a particularly good candidate for checkpoint blockade immunotherapy. Cancer Discov. 5, 16-18 (2015).

10. Le, D. T. \& Durham, J. N. Mismatch repair deficiency predicts response of solid tumors to PD-1 blockade. Science 357, 409-413 (2017).

11. Tsilimigras, M. C., Fodor, A. \& Jobin, C. Carcinogenesis and therapeutics: the microbiota perspective. Nat. Microbiol. 2, 17008 (2017).

12. Sears, C. L. \& Garrett, W. S. Microbes, microbiota, and colon cancer. Cell Host Microbe 15, 317-328 (2014).

13. De Sousa, E. M. F. et al. Poor-prognosis colon cancer is defined by a molecularly distinct subtype and develops from serrated precursor lesions. Nat. Med. 19, 614-618 (2013)

14. Sadanandam, A. et al. A colorectal cancer classification system that associates cellular phenotype and responses to therapy. Nat. Med. 19, 619-625 (2013).

15. Guinney, J. et al. The consensus molecular subtypes of colorectal cancer. Nat. Med. 21, 1350-1356 (2015).

16. Isella, C. et al. Stromal contribution to the colorectal cancer transcriptome. Nat. Genet. 47, 312-319 (2015).

17. Calon, A. et al. Stromal gene expression defines poor-prognosis subtypes in colorectal cancer. Nat. Genet. 47, 320-329 (2015).

18. Vellinga, T. T. et al. Collagen-rich stroma in aggressive colon tumors induces mesenchymal gene expression and tumor cell invasion. Oncogene 35, 5263-5271 (2016).

19. Tredan, O., Galmarini, C. M., Patel, K. \& Tannock, I. F. Drug resistance and the solid tumor microenvironment. J. Nat/ Cancer Inst. 99, 1441-1454 (2007).

20. Wang, L. et al. EMT- and stroma-related gene expression and resistance to PD-1 blockade in urothelial cancer. Nat. Commun. 9, 3503 (2018).

21. Isella, C. et al. Selective analysis of cancer-cell intrinsic transcriptional traits defines novel clinically relevant subtypes of colorectal cancer. Nat. Commun. 8, 15107 (2017).

22. Cancer Genome Atlas Network. Comprehensive molecular characterization of human colon and rectal cancer. Nature 487, 330-337 (2012).

23. Marisa, L. et al. Gene expression classification of colon cancer into molecular subtypes: characterization, validation, and prognostic value. PLoS Med. 10, e1001453 (2013).

24. Rubio-Perez, C. et al. In silico prescription of anticancer drugs to cohorts of 28 tumor types reveals targeting opportunities. Cancer Cell 27, 382-396 (2015).

25. Hoshida, Y. Nearest template prediction: a single-sample-based flexible class prediction with confidence assessment. PLOS ONE 5, e15543 (2010).

26. Heerboth, S. et al. EMT and tumor metastasis. Clin. Transl. Med. 4, 6 (2015).

27. Laiho, P. et al. Serrated carcinomas form a subclass of colorectal cancer with distinct molecular basis. Oncogene 26, 312-320 (2007).

28. He, J. et al. Qualitative transcriptional signature for predicting pathological response of colorectal cancer to FOLFOX therapy. Cancer Sci. 111, 253-265 (2020).

29. Tong, M. et al. Identifying clinically relevant drug resistance genes in druginduced resistant cancer cell lines and post-chemotherapy tissues. Oncotarget $\mathbf{6}$, 41216-41227 (2015)

30. Alberts, S. R. et al. Effect of oxaliplatin, fluorouracil, and leucovorin with or without cetuximab on survival among patients with resected stage III colon cancer: a randomized trial. JAMA 307, 1383-1393 (2012).

31. Bertotti, A. et al. The genomic landscape of response to EGFR blockade in colorectal cancer. Nature 526, 263-267 (2015).

32. Khambata-Ford, S. et al. Expression of epiregulin and amphiregulin and K-ras mutation status predict disease control in metastatic colorectal cancer patients treated with cetuximab. J. Clin. Oncol. 25, 3230-3237 (2007).

33. Zanella, E. R. et al. IGF2 is an actionable target that identifies a distinct subpopulation of colorectal cancer patients with marginal response to anti-EGFR therapies. Sci. Transl. Med. 7, 272 ra212 (2015).

34. Bray, S. M. et al. Genomic characterization of intrinsic and acquired resistance to cetuximab in colorectal cancer patients. Sci. Rep. 9, 15365 (2019).

35. Gray, R. G. et al. Validation study of a quantitative multigene reverse transcriptase-polymerase chain reaction assay for assessment of recurrence risk in patients with stage II colon cancer. J. Clin. Oncol. 29, 4611-4619 (2011).

36. Clark-Langone, K. M., Sangli, C., Krishnakumar, J. \& Watson, D. Translating tumor biology into personalized treatment planning: analytical performance characteristics of the Oncotype DX ${ }^{\circledR}$ Colon Cancer Assay. BMC Cancer 10, 691 (2010). 
37. Van Cutsem, E., Cervantes, A., Nordlinger, B. \& Arnold, D. Metastatic colorectal cancer: ESMO Clinical Practice Guidelines for diagnosis, treatment and follow-up. Ann. Oncol. 25, iii1-iiig (2014).

38. Goldberg, R. M. Therapy for metastatic colorectal cancer. Oncologist 11, 981-987 (2006).

39. Sonagara, D. \& Badheka, S. Comparison of basic clustering algorithms. Int. J. Computer Sci. Mob. Comput., 3, 58-61 (2014).

40. Ogino, S., Kawasaki, T., Kirkner, G. J., Loda, M. \& Fuchs, C. S. CpG island methylator phenotype-low (CIMP-low) in colorectal cancer: possible associations with male sex and KRAS mutations. J. Mol. Diagn. 8, 582-588 (2006).

41. Liang, J. J., Bissett, I., Kalady, M., Bennet, A. \& Church, J. M. Importance of serrated polyps in colorectal carcinogenesis. ANZ J. Surg. 83, 325-330 (2013).

42. Sargent, D. J. et al. Defective mismatch repair as a predictive marker for lack of efficacy of fluorouracil-based adjuvant therapy in colon cancer. J. Clin. Oncol. 28, 3219-3226 (2010).

43. Ribic, C. M. et al. Tumor microsatellite-instability status as a predictor of benefit from fluorouracil-based adjuvant chemotherapy for colon cancer. N. Engl. J. Med. 349, 247-257 (2003).

44. Burns, M. B. et al. Discrete mutations in colorectal cancer correlate with defined microbial communities in the tumor microenvironment. Preprint at bioRxiv https://doi.org/10.1101/090795 (2016).

45. Castellarin, M. et al. Fusobacterium nucleatum infection is prevalent in human colorectal carcinoma. Genome Res. 22, 299-306 (2012).

46. Burns, M. B., Lynch, J., Starr, T. K., Knights, D. \& Blekhman, R. Virulence genes are signature of the microbiome in the colorectal tumor microenvironment. Genome Med. 7, 55 (2015).

47. Lennard, K. S., Goosen, R. W. \& Blackburn, J. M. Bacterially-associated transcriptional remodelling in a distinct genomic subtype of colorectal cancer provides a plausible molecular basis for disease development. PloS ONE 11, e0166282 (2016).

48. Bonnet, M. et al. Colonization of the human gut by E. coli and colorectal cancer risk. Clin. Cancer Res. 20, 859-867 (2014).

49. Shannon, B. A., Garrett, K. L. \& Cohen, R. J. Links between Propionibacterium acnes and prostate cancer. Future Oncol. 2, 225-232 (2006).

50. Gautier, L., Cope, L., Bolstad, B. M. \& Irizarry, R. A. Affy-analysis of Affymetrix GeneChip data at the probe level. Bioinformatics 20, 307-315 (2004).

51. Calon, A. et al. Dependency of colorectal cancer on a TGF-beta-driven program in stromal cells for metastasis initiation. Cancer Cell 22, 571-584 (2012).

52. Del Rio, M. et al. Gene expression signature in advanced colorectal cancer patients select drugs and response for the use of leucovorin, fluorouracil, and irinotecan. J. Clin. Oncol. 25, 773-780 (2007).

53. Ritchie, M. E. et al. limma powers differential expression analyses for RNAsequencing and microarray studies. Nucleic Acids Res. 43, e47 (2015).

54. Jorissen, R. N. et al. Metastasis-associated gene expression changes predict poor outcomes in patients with dukes stage B and C colorectal cancer. Clin. Cancer Res. 15, 7642-7651 (2009)

55. Smith, J. J. et al. Experimentally derived metastasis gene expression profile predicts recurrence and death in patients with colon cancer. Gastroenterology 138 958-968 (2010)

56. de Sousa, E. M. F. et al. Methylation of cancer-stem-cell-associated Wnt target genes predicts poor prognosis in colorectal cancer patients. Cell Stem Cell $\mathbf{9}$, 476-485 (2011).

57. Laibe, S. et al. A seven-gene signature aggregates a subgroup of stage II colon cancers with stage III. Omics 16, 560-565 (2012)

58. Johnson, W. E., Li, C. \& Rabinovic, A. Adjusting batch effects in microarray expression data using empirical Bayes methods. Biostatistics 8, 118-127 (2007).

59. Tsuji, S. et al. Potential responders to FOLFOX therapy for colorectal cancer by Random Forests analysis. Br. J. Cancer 106, 126-132 (2012).

60. Li, S., Lu, X., Chi, P. \& Pan, J. Identification of HOXB8 and KLK11 expression levels as potential biomarkers to predict the effects of FOLFOX4 chemotherapy. Future Oncol. 9, 727-736 (2013).

61. Okita, A. et al. Consensus molecular subtypes classification of colorectal cancer as a predictive factor for chemotherapeutic efficacy against metastatic colorectal cancer. Oncotarget 9, 18698-18711 (2018).

62. Del Rio, M. et al. Molecular subtypes of metastatic colorectal cancer are associated with patient response to irinotecan-based therapies. Eur. J. Cancer 76, 68-75 (2017).

63. $\mathrm{Li}, \mathrm{H}$. et al. Reference component analysis of single-cell transcriptomes elucidates cellular heterogeneity in human colorectal tumors. Nat. Genet. 49, 708-718 (2017).

64. Puram, S. V. et al. Single-cell transcriptomic analysis of primary and metastatic tumor ecosystems in head and neck cancer. Cell 171, 1611-1624.e1624 (2017).

65. Tirosh, I. et al. Dissecting the multicellular ecosystem of metastatic melanoma by single-cell RNA-seq. Science 352, 189-196 (2016).

66. Chung, W. et al. Single-cell RNA-seq enables comprehensive tumour and immune cell profiling in primary breast cancer. Nat. Commun. 8, 15081 (2017).
67. Li, W. V. \& Li, J. J. An accurate and robust imputation method sclmpute for singlecell RNA-seq data. Nat. Commun. 9, 997 (2018).

68. McCarthy, D. J., Campbell, K. R., Lun, A. T. \& Wills, Q. F. Scater: pre-processing, quality control, normalization and visualization of single-cell RNA-seq data in R. Bioinformatics 33, 1179-1186 (2017).

69. Kim, D., Langmead, B. \& Salzberg, S. L. HISAT: a fast spliced aligner with low memory requirements. Nat. Methods 12, 357-360 (2015).

70. Liao, Y., Smyth, G. K. \& Shi, W. featureCounts: an efficient general purpose program for assigning sequence reads to genomic features. Bioinformatics $\mathbf{3 0}$ 923-930 (2014).

71. Thorsson, V. et al. The immune landscape of cancer. Immunity $\mathbf{4 8 , 8 1 2 - 8 3 0 . e 8 1 4}$ (2018).

72. Kakushadze, Z. \& Yu, W. *K-means and cluster models for cancer signatures. Biomol. Detect. Quantif. 13, 7-31 (2017).

73. Dubey, A. K., Gupta, U. \& Jain, S. Analysis of k-means clustering approach on the breast cancer Wisconsin dataset. Int. J. Computer Assist. Radiol. Surg. 11, 2033-2047 (2016).

74. Tibshirani, R., Walther, G. \& Hastie, T. Estimating the number of clusters in a data set via the gap statistic. J. R. Stat. Soc. Ser. B 63, 411-423 (2001).

75. Bindea, G. et al. ClueGO: a Cytoscape plug-in to decipher functionally grouped gene ontology and pathway annotation networks. Bioinformatics 25, 1091-1093 (2009).

76. Merlos-Suarez, A. et al. The intestinal stem cell signature identifies colorectal cancer stem cells and predicts disease relapse. Cell Stem Cell 8, 511-524 (2011).

77. Kosinski, C. et al. Gene expression patterns of human colon tops and basal crypts and BMP antagonists as intestinal stem cell niche factors. Proc. Natl Acad. Sci. USA 104, 15418-15423 (2007)

78. Loboda, A. et al. EMT is the dominant program in human colon cancer. BMC Med. Genomics 4, 9 (2011).

79. Graudens, E. et al. Deciphering cellular states of innate tumor drug responses. Genome Biol. 7, R19 (2006).

80. Schutte, M. et al. Molecular dissection of colorectal cancer in pre-clinical models identifies biomarkers predicting sensitivity to EGFR inhibitors. Nat. Commun. 8 14262 (2017).

81. Newman, A. M. et al. Robust enumeration of cell subsets from tissue expression profiles. Nat. Methods 12, 453-457 (2015).

82. Becht, E. et al. Estimating the population abundance of tissue-infiltrating immune and stromal cell populations using gene expression. Genome Biol. 17, 218 (2016).

83. Hanzelmann, S., Castelo, R. \& Guinney, J. GSVA: gene set variation analysis for microarray and RNA-seq data. BMC Bioinformatics 14, 7 (2013).

84. Barbie, D. A. et al. Systematic RNA interference reveals that oncogenic KRAS driven cancers require TBK1. Nature 462, 108-112 (2009).

85. Mariathasan, S. et al. TGFbeta attenuates tumour response to PD-L1 blockade by contributing to exclusion of T cells. Nature 554, 544-548 (2018).

86. Jiang, P. et al. Signatures of $\mathrm{T}$ cell dysfunction and exclusion predict cancer immunotherapy response. Nat. Med. 24, 1550-1558 (2018).

87. Givechian, K. B. et al. Identification of an immune gene expression signature associated with favorable clinical features in Treg-enriched patient tumor samples. NPJ Genom. Med. 3, 14 (2018).

88. Ayers, M. et al. IFN-gamma-related mRNA profile predicts clinical response to PD1 blockade. J. Clin. Investig. 127, 2930-2940 (2017).

89. Hugo, W. et al. Genomic and transcriptomic features of response to anti-PD-1 therapy in metastatic melanoma. Cell 165, 35-44 (2016).

90. O'Connell, M. J. et al. Relationship between tumor gene expression and recurrence in four independent studies of patients with stage II/III colon cancer treated with surgery alone or surgery plus adjuvant fluorouracil plus leucovorin. J. Clin. Oncol. 28, 3937-3944 (2010).

91. Kostic, A. D. et al. PathSeq: software to identify or discover microbes by deep sequencing of human tissue. Nat. Biotechnol. 29, 393-396 (2011).

92. Bullman, S. et al. Analysis of Fusobacterium persistence and antibiotic response in colorectal cancer. Science 358, 1443-1448 (2017).

93. Zhu, X. et al. Metadata record for the manuscript: a tumor microenvironment specific gene expression signature predicts chemotherapy resistance in colorectal cancer patients. figshare https://doi.org/10.6084/m9.figshare.13027715 (2020).

94. Gene Expression Omnibus. The molecular subtype in colorectal cancer. https:// identifiers.org/geo:GSE158559 (2020).

\section{ACKNOWLEDGEMENTS}

The authors are grateful for all the subjects who participated in the study. This work was supported by grants from the National Natural Science Foundation of China Nos. \#81874159, 82073115, 31371273, 81871901, and 81602518; The Program for Professor of Special Appointment (2015 Youth Eastern Scholar No. QD2015003 and Eastern Scholar No. 201268) at Shanghai Institutions of Higher Learning; Shanghai Municipal Education Commission-Gaofeng Clinical Medicine Grant Support (Nos. 20161309 and 20152512); and Chenxing Project of Shanghai Jiao Tong University to 
H.C. 2018 Changning District Medical and Health Research Project (No. CNKW2018Y11) to X.T.

\section{AUTHOR CONTRIBUTIONS}

X.Z., X.T., and L.J. contributed equally to this work. X.Z., X.T., L.J., J.-Y.F., J.H., and H.C. participated in the design and performance of the study. X.Z., X.T., L.J., Y.C., C.Y., C.S., Y.H., J.W., J.-Y.F., J.H., and H.C. participated in analysis and interpretation of the data. X.Z., X.T., L.J., X.Z., C.S., and H.C. performed statistical analysis. The manuscript was draft by X.Z., X.T., L.J., and H.C. and reviewed by all authors. All authors read and approved the final manuscript.

\section{COMPETING INTERESTS}

The authors declare no competing interests.

\section{ADDITIONAL INFORMATION}

Supplementary information The online version contains supplementary material available at https://doi.org/10.1038/s41698-021-00142-x.

Correspondence and requests for materials should be addressed to J.-Y.F., J.H. or H.C.
Reprints and permission information is available at http://www.nature.com/ reprints

Publisher's note Springer Nature remains neutral with regard to jurisdictional claims in published maps and institutional affiliations.

Open Access This article is licensed under a Creative Commons Attribution 4.0 International License, which permits use, sharing, adaptation, distribution and reproduction in any medium or format, as long as you give appropriate credit to the original author(s) and the source, provide a link to the Creative Commons license, and indicate if changes were made. The images or other third party material in this article are included in the article's Creative Commons license, unless indicated otherwise in a credit line to the material. If material is not included in the article's Creative Commons license and your intended use is not permitted by statutory regulation or exceeds the permitted use, you will need to obtain permission directly from the copyright holder. To view a copy of this license, visit http://creativecommons. org/licenses/by/4.0/.

(c) The Author(s) 2021 